\title{
Die Entdeckung der Welt-Kunst in der Frühen Neuzeit: Bildphantasien und Bilderproduktion der Vier Erdteile
}

\author{
Ulrich Pfisterer
}

\section{Standpunkte, Blickwechsel und 'ethnographischer Bildvergleich'}

Wenige andere Bildwerke provozierten in der Frühen Neuzeit so dezidierte Aussagen über die Standpunkte und Blickweisen auf die Welt wie Karten. Von einem Aufeinandertreffen unterschiedlicher kartographischer Traditionen und Positionen berichtete etwa ausführlich der italienische Jesuiten-Missionar Matteo Ricci. Die Aufzeichnungen seines langjährigen China-Aufenthaltes wurden 1615 von Nicolas Trigault erstmals auf Latein herausgegeben und dann sofort ins Französische, Italienische und Deutsche übersetzt - sie dürften damit zu den am besten verfügbaren Nachrichten über Asien gehört haben:

Dann als einer under den Verschnitnen [Hofbeamten] dem König [dem Kaiser von China] die Landtafel [die von Ricci konzipierte, gedruckte Weltkarte] presentiert/darob deß P. Matthei als Autors namen gestanden / hat er begert $/ \mathrm{man}$ soll jhme 12. exemplar auff Taffet trucken/so er und er seinen Söhnen/und andern befreundten im Palast außtheiln wöllen: [...]. Der Pater hett jhms zwar langst gern verehrt/da er sich nicht besorgt/Er wurde es für ein verachtung seines Reichs halten/das es diß Orts für einen kleinen Particul deß Erdbodens angegeben wird/da doch er und die seinige biß dahin vermeint/es seye weit der größte theil desselbigen. Das es aber ein vergebne sorg gewest/ hat dißmal der augenschein zu erkennen gegeben/unnd der König einen hohen verstand erzeigt/inn dem er nicht darfür halten wöllen/das die warheit seinem Reich verkleinerlich sey. ${ }^{2}$

Exemplare dieser berühmten „Karte der unzähligen Länder der Welt", die Ricci 1584 in China hatte drucken lassen - von der sich aber wohl nur Exemplare einer Wiederauflage von 1602 erhalten haben (Abb. 1) - zeigen, dass die Situation doch komplizierter gewesen war: Denn tatsächlich wich die

1 Der Beitrag stellt eine erweiterte Fassung von „Idole und Ideale in der Kunst der Frühen Neuzeit - oder: Macht und Relativität der Phantasie", in: Effinger/Logemann / Pfisterer 2012, 93-105 dar.

2 Trigault 1617, $480 \mathrm{f}$. (Kap. xvi). 


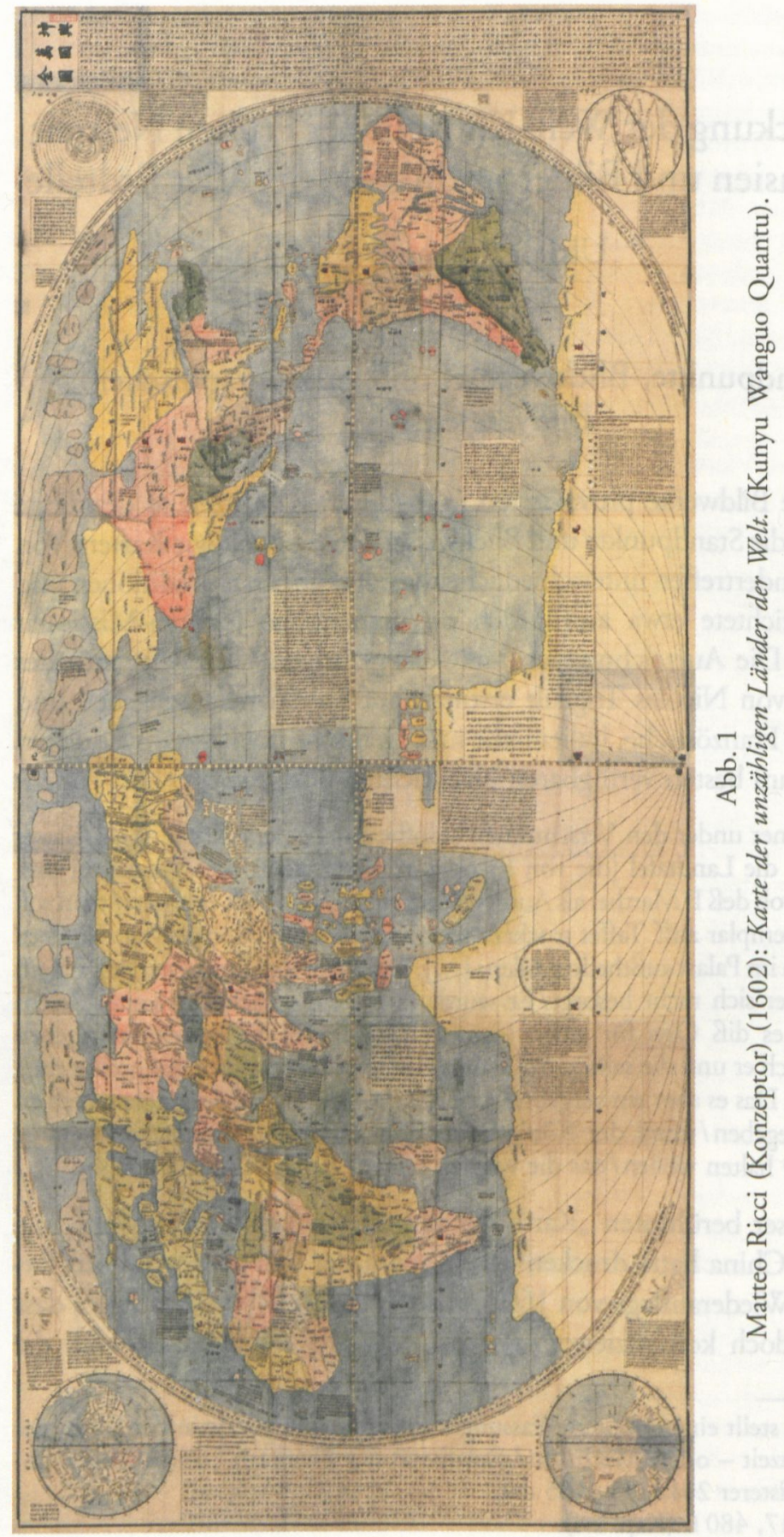


Karte des Missionars zwar fundamental von den bis dato kursierenden chinesischen Darstellungen des 'Reichs der Mitte' ab, die sich (etwas verkürzt gesagt) auf ein annähernd rundes China im Zentrum konzentrierten. Aber Ricci verschob doch im Unterschied zur europäischen Kartographie der damaligen Zeit China von einer Randstellung ganz im Osten entscheidend hin zur Mitte. Dieses Vorgehen fügt sich in die umfassender verfolgte Strategie der Jesuiten ein, über Kompromisse die europäische den 'fremden' Blickweisen zu vermitteln und zumindest teilweise auf die kulturellen Eigenheiten der fremden Völker einzugehen, um ihnen die Bekehrung zum Christentum möglichst leicht zu machen - eine allerdings bald so heftig umstrittene 'Akkomodationsmethode', dass etwa um die Mitte des 17. Jahrhunderts Giovanni Battista Riccioli und Daniello Bartoli das Vorgehen ihres Mitbruders Ricci rechtfertigen mussten. ${ }^{3}$ In diesem Sinne hatte Ricci China zwar in für chinesische Augen ungewohnt 'wahren' Größenrelation zum Rest der Welt dargestellt, aber immerhin in einer Position nahe der Mitte belassen. ${ }^{4}$ Andere visuelle Verfahren - etwa die Adaptation von Nadals Evangelicae Historiae Imagines, dem jesuitischen 'Bilder-Lehrbuch' schlechthin, in allen Erdteilen oder dann später die von dem Italiener Giovanni Castiglione gemalten chinesischen Herrscherbilder - ließen sich hier anfügen. ${ }^{5}$ Andererseits erinnert das Beispiel chinesischer Bildproduktion auch besonders intensiv daran, dass ein 'kolonialer Blick' nicht nur auf europäischer Seite existierte und also das Aufeinandertreffen von europäischem 'Orientalismus' und asiatischem 'Okzidentalismus' eine besondere Herausforderung darstellt. ${ }^{6}$

So offensichtlich sich bei diesen Beispielen unterschiedliche Blick- und Darstellungsweisen überlagerten: Die 'Wahrheit' und damit Superiorität der europäischen Darstellungstradition stand für Ricci und sein europäisches Publikum in diesen Fällen außer Frage. Eine andere, auf den ersten Blick unerwartete Gruppe von Bildwerken veranlasste dagegen nicht nur den Vergleich von europäischen und außer-europäischen Erzeugnissen, sondern

3 Zu Ricci und den Jesuiten in China zuletzt umfassend Hsia 2009; Fontana 2010; Hsia 2010.

4 Zur chinesischen Kartographie etwa Hostetler 2001; Caterino 2008 und Klüser 2009.- $\mathrm{Zu}$ vergleichbaren Vorgängen und Visualisierungen in Japan, wo die 'Vermittlungsleistung' zwischen japanischer und europäischer Kartographie von einheimischen Malern geleistet zu sein scheint, siehe Mochizuki 2010.

5 Zur Verbreitung von Nadals Imagines in den Erdteilen vgl. etwa Bosc-Tiessé 2004; Lazure 2007; Borao Mateo 2010; zu Castiglione vgl. Pirazzoli-T'Serstevens 2007; vgl. übergreifend auch Purtle 2010.

6 Vgl. die Beispiele bei Deal/Hostetler 2006; zu 'Einflüssen' chinesischer Malerei auf Europa vgl. etwa Ledderose 1991. 
provozierte eine tiefgreifende Erschütterung des visuellen und ästhetischen Kanons in Europa und führte letztlich zu einer zumindest ansatzweisen Einsicht in die Relativität aller, auch der eigenen Bildproduktion und ästhetischen Normen. Die Rede ist von den heidnischen 'Idolen', wie sie ab dem 16. Jahrhundert verstärkt in Europa bekannt wurden und bei denen man zunächst annehmen möchte, dass sie die schärfste Ablehnung und das größte Unverständnis erfuhren. Die Herausforderung dieser 'Götzenbilder' für das europäische Denken der Frühen Neuzeit soll im Folgenden skizziert werden. Dabei wird sich nicht nur zeigen, dass die visuelle Alterität dieser fremden Bildwerke neue Formen der Bilddokumentation verlangte. Erkennbar wird auch ein zur frühneuzeitlichen Kunstliteratur im engeren Sinne alternativer oder komplementärer Kunstdiskurs, der nicht auf den Kriterien 'Naturnachahmung' und (normative) 'Schönheit' basierte, sondern auf eine Entwicklungsgeschichte menschlicher Bild-Phantasien und damit Schönheitsvorstellungen über 'alle Zeiten und Völker' hinweg abzielt. Insofern sich hier die Möglichkeit einer ästhetischen Wertschätzung sui generis (also über die Wertschätzung der Exotica als curiosa und rara hinaus) andeutet, scheint es berechtigt, von einer 'Entdeckung der Weltkunst' in der Frühen Neuzeit zu sprechen.

Die europäische Wahrnehmung Chinas und seiner Bildwerke liefert auch hierfür ein besonders schlagendes Beispiel des Nebeneinanders unterschiedlicher, teils konkurrierender Vorstellungen und Positionen. So konnte Grimmelshausen 1668/1669 in seinem Simplicissimus scharf die Obsession eines vermeintlichen 'Kunstliebhabers', der die heidnischen Bilder über die christlichen stellt, verurteilen:

Er [der Sammler] aber sagte, ich irre mich; das Chineser Gemäld wäre rarer und dahero auch köstlicher; er wolle es nicht um zehen solcher Ecce-Homo manglen. Ich antwortet: Herr! Ist Euer Herz wie Euer Mund? Er sagte: Ich versehe michs. Darauf sagte ich: So ist auch Euers Herzen Gott derjenige, dessen Conterfait Ihr mit dem Mund bekennet, das köstlichste zu sein. - Phantast, sagte jener, ich ästimiere die Rarität.

Annähernd gleichzeitig wird Joachim von Sandrart zwar ebenfalls kritisieren:

Die Chineser lieben [Bildereyen]/haben aber keine Wissenschaft von den Oelfarben/und mahlen einfältig/nur in Profil oder Umriß. ${ }^{8}$

Immerhin widmet er aber erstmals in der Geschichte europäischer Kunstliteratur der „Chineser Mahlerey" ein eigenes Kapitel seiner Teutschen Academie

7 Grimmelshausen 1977, $73 \mathrm{f}$.

8 Sandrart 1675-1679, Teil I, Buch iii, Kap. 16, 100-103. 
und gesteht auch zu, die Chinesen seien „ziemlich erfahren“ in Malerei und Skulptur, „[s]onderlich bedienen sie sich derselben in ihren Tempeln: alda sie viel Abgötter haben“, und die mangelnde Naturbeobachtung und Perspektivkenntnisse ließen sich angesichts des „ihnen beywohnenden auserlesnen Verstand[es] " unter Anleitung europäischer Maler leicht verbessern. Zudem bildet Sandrart erstmals - am Ende seiner Porträtreihe italienischer Künstler mit „Higiemonte“ das Bildnis eines nicht-europäischen „Indianer M[alers]“ ab (Abb. 2). ${ }^{9}$ Bereits ein knappes Jahrhundert zuvor freilich war der Jesuit Juan González de Mendoza in seiner Einschätzung chinesischer Kunst noch weit über Sandrart hinaus gegangen. In seinen erstmals 1585 auf Spanisch, 1589 und 1598 in deutscher Übersetzung gedruckten Historien und Bericht von dem newlicher Zeit erfundenen Königreich China liest man ein uneingeschränktes Lob der fremden Malerei (allerdings scheint die Darstellung von Menschen ausgenommen):

Sie [die Chinesen] sind auch kunstreiche un[d] vortreffliche Mahler/von Laubwerck/Gevögel und aller Art wildes gewächs/wie man wol sehen kan an den Kasten unnd Tafeln/die aus dem Lande zu uns kommen/deren eine ich gesehen/die der Häuptman Ribera/[...]/im Jar 1582. gen Lyßbona bracht/von deren Vortreffligkeit und Schönheit ist dieses genug gesagt/daß sie nit als andere/so sie sehen/sich darob verwundern möchten/sondern auch (welches selten geschicht) den König aus Hispanien selbst ein wunderbarlich Werck seyn bedauchte/und noch andere in der Kunst berümbte Leut auch. ${ }^{10}$

Gerühmt wird auch die chinesische Baukunst, die es offenbar mit der (römischen) Antike aufnehmen kann-selbst ein Architekturtraktat sei nach Europa gesandt worden:

Die [...] Gassen [chinesischer Städte] sind mit viel Triumphbogen zugericht/in gleicher weiter von einander erbawet/darvon sie so hübsch anzusehen/als im [m] er müglich ist. Denn solche Bogen von guten Wercksteinen mit mancherley schönen lustigen Bildwecken gezieret sind/welche den Römischen Antiquiteten

9 Sandrart 1675-1679, Teil II, Buch ii, Taf. T (nach 200); die kurzen Informationen zu „Higiemondo" auf 100. - Ein ausführlicher, lobender Kommentar zu den südamerikanischen (Feder-)Malern findet sich bereits 1560 bei Guevara 1948, 342 f., allerdings im Kapitel zur Malerei der alten Ägypter, von denen laut Guevara die "Indios occidentales" abstammten und daher auch „unas mismas imaginaciones“ hätten. Zu den Manuskript gebliebenen Überlegungen des Francisco de Holanda siehe Deswartes Rose 1985.

10 González de Mendoza 1598, 28; vgl. auch 29 f. zu Porzellanen; 72 zur Verehrung von Königsbildern. - Zu anderen chinesischen Malereien in einer Kunstkammer dieser Zeit siehe Whitfield 1976, zu japanischen Lackkasten siehe Schweizer u.a. 2011. 


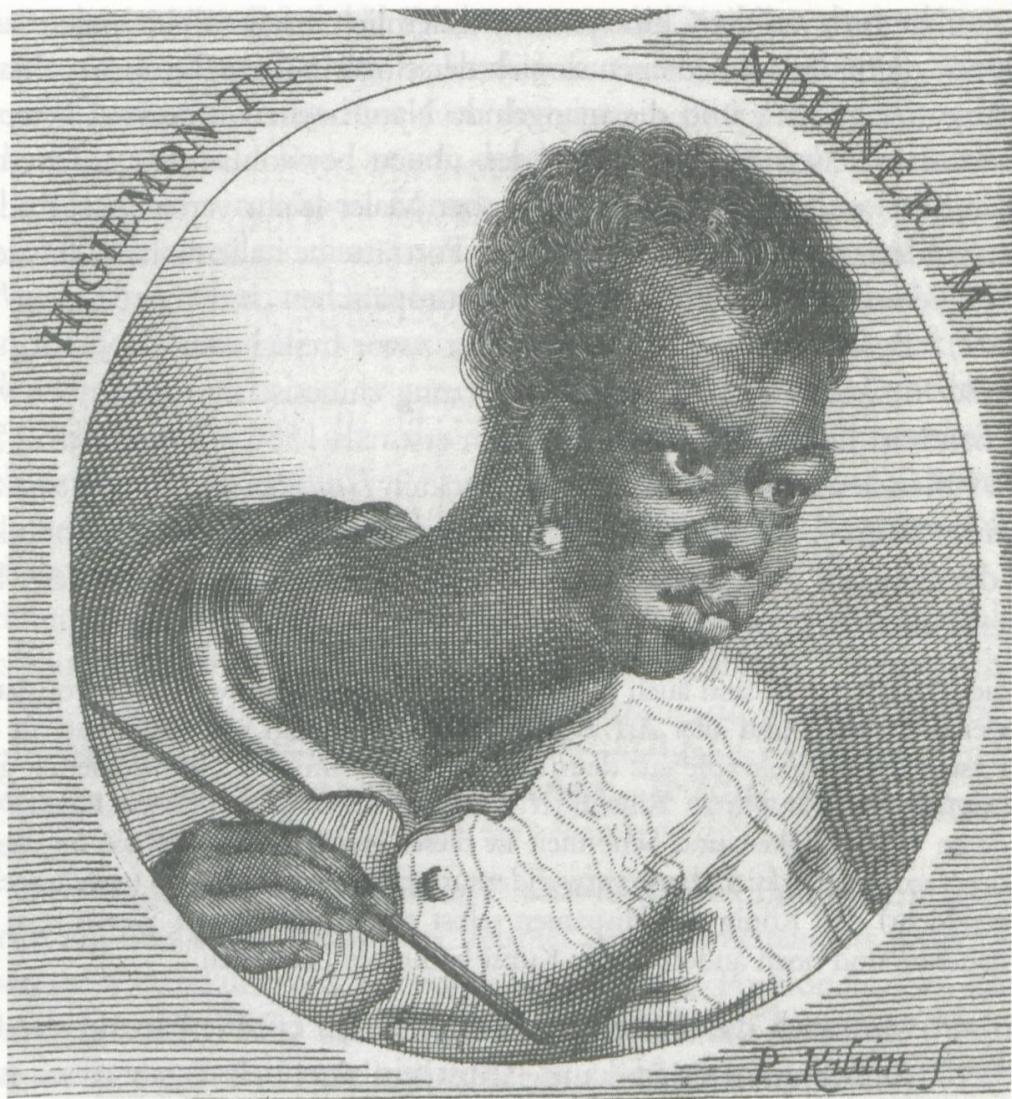

Abb. 2

Der „Indianer Maler“ Higiemonte, in: Sandrart, Joachim von (1675-1679): Teutsche Academie der edlen Bau-, Bild- und Mablerey-Künste. 2 Bde. Nürnberg. Teil II, Buch ii, Taf. T (nach S. 200).

sich vergleichen/mit schönen Perspectivischen und andern kunstreichen Stücken. ${ }^{11}$

Vor allem aber dienen die Bildwerke dem Missionar auch als Geschichtszeugnisse einer längst vergangenen Phase der chinesischen Kultur-

11 González de Mendoza 1598, 21; das folgende Kapitel I, 9 handelt ausschließlich von den wunderbaren Bauwerken in China; zum Architekturtraktat 133-vgl. dagegen einen Brief des Johannes Schreck S.J. von 1622 (zitiert nach Walravens 1987, 34): „Wir sahen dort [in Macao] ungefährt 100 schöne Triumphbögen aus Steinquadern, aber nicht so schön wie die römischen. Ich hätte sie gezeichnet, wäre es uns erlaubt, öffentlich auszugehen." 
und Religionsgeschichte. Denn die ikonographischen Parallelen zwischen den chinesischen Götzenbildern (etwa eine Frau mit Kind auf dem Arm) und den christlich-katholischen Bildern des 'wahren Glaubens' ließen sich nur als Relikte aus der Zeit verstehen, als der Heilige Thomas Asien zu bekehren versuchte. In der Folgezeit breitete sich zwar die Idolatrie in Asien aus, allein bestimmte Elemente seien zunehmend unverstanden weiter bis ins späte 16. Jahrhundert tradiert worden. ${ }^{12}$ Dieses Argument des 'ethnographischen Bildvergleichs' (dessen Geschichte bislang nicht umfassend untersucht ist, so dass sich nur vermuten lässt, dass González de Mendoza und die Jesuiten dabei eine Schlüsselposition einnehmen ${ }^{13}$ ) wird im Weiteren eine zentrale Rolle spielen. Allerdings bleibt der Blick hierfür nicht auf China beschränkt. Eine letzte entscheidende Prämisse dieses Beitrags ist, dass - anders als die moderne Forschung, die sich häufig auf eine geographische Region konzentriert ${ }^{14}$ - die frühneuzeitlichen Auseinandersetzungen mit Idolen alle zu diesem Zeitpunkt in Europa bekannten vier Erdteile vergleichend und synthetisierend in den Blick nehmen und auch erst in der Zusammenschau aller dieser Äußerungen die hier postulierte alternative 'Geschichte der Welt-Kunst' erkennbar wird.

\section{Es werde Gott ...}

Einst war ich nur ein Stumpf vom Feigenbaum, Holz ohne Nutzen./ Da der Meister in Zweifel, ob eine Bank oder ein Priapus draus zu machen sei, / Entschied er sich für den Gott. Darum bin ich ein Gott [...].

Jedes Götterbild verdankt seine Gestalt menschlicher Phantasie und Kunstfertigkeit. Horaz hat diesen Gedanken in seiner Satire 1, 8 besonders wirkmächtig formuliert: Es ist die Entscheidung des Schnitzers, ob aus dem einen Holzklotz ein schlichter Gebrauchsgegenstand wie ein Tisch oder aber ein Objekt kultischer Verehrung wird. Auch bei anderen antiken Autoren wie Cicero, Lukrez und-ebenfalls besonders intensiv rezipiert dem vermeintlichen Hermes Trismegistus finden sich ähnliche Äußerun-

12 Vgl. González de Mendoza 1598, 31 f.-Zur Geschichte dieser Vorstellungen auch Rogers 1962.

13 Zur Geschichte ethnologischer Vergleiche Pagden 1986; zu vergleichenden Verfahren der Kunstwissenschaft im 19. und 20. Jahrhundert zuletzt Rees 2012.

14 Vgl. dagegen bereits Baily 1999 oder Kraus/Ottomeyer 2007. 
gen. ${ }^{15}$ Noch über Horaz hinaus geht dabei etwa Xenophanes, indem er die Anthropozentrik der menschlichen Phantasie-Produktion im Hinblick auf das Göttliche offen legt:

Wenn auch Kühe und Pferde oder Löwen Hände hätten und mit diesen ihren Händen zeichnen oder insgesamt das tun könnten, was Menschen mit ihren Händen tun können, dann würden Pferde ihre Götter in Pferdegestalt darstellen und die Kühe die ihren in Kuhgestalt $[\ldots]{ }^{16}$

Das Christentum, das seine zunächst ablehnende Haltung gegenüber Bildwerken im Laufe der ersten nachchristlichen Jahrhunderte weithin aufgegeben hatte, reagierte auf diese Vorstellungen mit einer zweigleisigen Legitimierungs-Strategie: Zum einen sollten zahlreiche Berichte und Wunder von angeblich 'wahren Abbildern' deren historische oder noch besser direkt durch Gott bezeugte Authentizität sicherstellen (notfalls ließ sich auch einfach auf Alter und lange Verehrungstradition verweisen). Zum anderen wurden verschiedene Grade der Verehrung von Bildwerken unterschieden, wobei-zumindest in der Theorie-diese nie direkt dem materiellen Objekt, sondern immer dem repräsentierten Prototypen galt. Allerdings bemühten auch die christlichen Theologen diese Götter(bilder) erschaffende menschliche Phantasie, freilich nur, um die Kultbilder anderer Religionen diffamieren zu können. So begründete etwa Origines in seinem Kommentar zum zweiten Gebot (2 Moses 20, 4) die Unterscheidung, wonach es zum einen fälschlich angebetete „Abbilder[...]“ real existierender Dinge (also etwa das Goldene Kalb) gebe, zum anderen noch eine Stufe schlimmer- „Götzen“, bei denen es sich um rein fiktive Produkte (durch den Teufel bzw. magische Praktiken) fehlgeleiteter Phantasien handelte, etwa Mischwesen aus tierischen und menschlichen Körperteilen. ${ }^{17}$

Nach dem byzantinischen Bilderstreit und den verstreuten mittelalterlichen Disputen über den Status von Bildern wurden dann insbesondere während der Glaubenskämpfe des 16. Jahrhunderts diese Fragen auf christliche Bildwerke angewandt: Dem altgläubigen Bildeinsatz konnte

15 Vgl. Cicero: De natura deorum, 1, 18-27 und 81-100; Lukrez, 3, 978-1023 und V, 1198-1203; Hermes Trismegistus: Asclepius, Kap. 8: „homo effector est deorum, qui in templis sunt"; Minucius Felix: Octavius, 23.

16 Diels/Kranz 1960, 21; vgl. etwa auch Epicharm bei Diogenes Laertios: Vitae philosophorum, 3, 16.

17 Dazu Ginzburg 1999; zum Weiteren Camille 1989; vgl. für den weiteren Kontext etwa Miller 2004 und für die Vorstellung von 'teuflischen Idolen der Phantasie' bis zur Frühen Neuzeit Steadman 2006. 
nun in unterschiedlicher Radikalität Idolatrie vorgeworfen werden, wobei manchmal das 'Von-Menschen-Gemacht-Sein' der Bilder überhaupt, manchmal speziell die von religiösen Belangen ablenkende, 'übertriebene Künstlichkeit', die mehr dem Ruhm des Künstlers als der Ehre Gottes diene, kritisiert wurden. Für Calvin etwa sind alle Bildwerke aufgrund ihrer Materialität und der durch sie notwendig affizierten Sinne „idola“. ${ }^{18}$ Und Luther, der mit seiner Bibelübersetzung 1534 den Begriff des 'Götzen’ im deutschsprachigen Raum populär machte, definierte diesen als trügerisches Phantasiegebilde, als all dasjenige, „woran der Mensch sein Herz hängt“ ${ }^{19}$ Francis Bacon wird um 1600 dann vier auf unser Denken insgesamt bezogene Formen täuschender ,idola“ unterscheiden. ${ }^{20}$ Auch das Horaz-Zitat konnte bei diesen Bilderdiskussionen wieder zum Einsatz kommen. ${ }^{21}$

Dass Gottesbilder Spiegel menschlicher Phantasien seien und vor allem auch, dass die überzeugende Verbildlichung durch einen Künstler entscheidend zu deren Erfolg beitragen könne, wurde spätestens mit der zweiten Hälfte des 17. Jahrhunderts weithin diskutiert. Eine brillante Entfaltung des Gedankens findet sich in Jean de la Fontaines Fabel vom Bildhauer und einer Statue des Jupiter (1668) - wo diese Chimären der Phantasie als besonders typisch für das Heidentum gelten:

Ein Marmorblock war so schön/Daß ein Bildhauer ihn kaufte. / Was wird, fragt er, mein Meißel daraus machen? Wird er Gott, Tisch oder Waschschüssel? / Er werde Gott. [...]. ${ }^{22}$

Im Kontext von Kunstliteratur im engeren Sinne steigerte Charles Perrault die Idee nochmals in seiner Parallele des anciens et des modernes (1688): Die antiken Künstler der besten Götterbilder seien in der Folge aus Ehrerbietung über ihre Leistung selbst gottgleich verehrt worden. ${ }^{23}$ Das eu-

18 Dazu Lecercle 2005, 157-159; vgl. ders. 1990.-Dass Idole allgemein mit Sinnlichkeit, Sexualität und "schimpflicher Nacktheit“ verbunden wurden, demonstriert gleich die erste mittelalterliche Darstellung heidnischer Götter zur Enzklopädie des Hrabanus Maurus: De rerum naturis, 15, 6, illuminiert in Montecassino um 1022, wo erneut Priapus / Belphegor mit erigiertem Geschlecht figuriert; dazu Himmelmann 1986, 8 und $13 \mathrm{f}$.

19 Dazu Prescher 2010.

20 Mit umfassender Verortung dazu Zittel 2002.

21 Etwa bei Hospinianus 1603, 157.

22 In La Fontaines Fables 9, 6; dazu die Analyse von Marin 2007.

23 Perrault 1668, Bd. 1, 189: „Il est encore à remarquer qu'il $\mathrm{y}$ avoit des récompenses extraordinaires attachées à la reüssite de ces sortes d'ouvrages, qu'il y alloit de donner des Dieux à des Nations entieres \& aux Princes mesmes de ces Nations; \& enfin que quand le Sculpteur avoit reüssi, il n'estoit gueres moins honoré que le 
hemeristische Erklärungsmodell, wonach große Menschen nach ihrem Tod vergöttlicht worden seien, wurde auf den Kult um 'göttliche Künstler' übertragen. ${ }^{24}$

Schließlich verweist der Abgesang auf die Idolatrie aus dem Jahr 1757 in nochmals neuer Wendung auf die Macht der Phantasie und zitiert dabei Horaz. Voltaire verfasste den Eintrag zu „Idole, Idolâtre, Idolâtrie" für den achten Band der Encyclopédie. ${ }^{25}$ Er bietet eine kurze Geschichte des Idolatrie-Gedankens, um auf dieser Basis zu argumentieren, dass keine einzige Glaubensrichtung von sich selbst behaupten würde, ihre Anhänger seien Götzendiener und Idol-Verehrer. Nicht nur die Götterbilder sind demnach der Phantasie entsprungen. Auch der Idolatrie-Vorwurf selbst basiert für Voltaire allein auf der unverständigen und abwertenden Projektion eigener, begrenzter Vorstellungen und Normen auf andere Religionen, eine kritische Einsicht, die sowohl für die inner-europäischen Glaubensstreitigkeiten wie für den Blick auf außereuropäische Religionen Geltung beanspruchen konnte. In letzter Konsequenz folgt aus dem Eintrag, dass alle materiellen Gottesbilder sich als gleichwertige Manifestationen verschiedener menschlicher Phantasien verstehen lassen.

\section{Schrecken und Schönheit der Götzenbilder}

Welche Rolle spielten in diesem Prozess nun die außereuropäischen Götterbilder, die im Laufe des 16. Jahrhunderts zunehmend in Reproduktionen bekannt wurden oder realiter in den Sammlungen zu betrachten waren? Gezeigt werden soll, dass diese Objekte und Bilder nicht nur in theologischen, historischen und ethnographischen Kontexten und Kategorien verstanden wurden. Vielmehr lassen sich zumindest einige Indizien dafür finden, dass die wachsende Einsicht in die Relativität der menschlichen Phantasien und Bildproduktion gerade angesichts dieser fremden

Dieu qui sortoit de ses mains. "Vgl. allerdings kritisch Perrault 1690, 31 -34, wo Skulptur gegen den pauschalen Vorwurf, idolatrischen Missbrauch zu begünstigen, verteidigt wird; der Fehler liege vielmehr in der Reaktion der Betrachter. - Zu diesen Diskussionen insgesamt Weinshenker 2008.

24 Der Gedanke findet sich ähnlich schon in Francisco de Hollandas De pintura antiga, Kap. 12, zitiert nach Olanda 2003, 42 f. Zu dessen Vorstellungen einer 'weltweiten Antike' im folgenden Kapitel, 44, vgl. Deswartes Rosa 1985.

25 Diderot/Alembert (Hrsg.): Encyclopédie, ou Dictionnaire raisonné des sciences, des arts et des métier, Bd. 8, Paris 1765, 501; der Text wurde dann auch in Voltaires Dictionnaire philosophique (1764) abgedruckt.-Dazu Rubiés 2006. 
'Götzen' mit einer Einsicht oder zumindest Ahnung in die Relativität und Vielfalt der menschlichen Wahrnehmung und ästhetischen Wertung insgesamt einher ging. ${ }^{26}$ Weitgehend akzeptiert waren dagegen $\mathrm{zu}$ diesem Zeitpunkt - sieht man von einer Phase der radikalen Idol-Zerstörung und des Ikonoklasmus während der Gegenreformation $\mathrm{ab}^{27}$ - die antik griechischen und römischen Götterstatuen, für die zumeist eine Trennung von verwerflichem, paganem Gehalt und rein formaler Betrachtung als überragende künstlerisch-ästhetische Vorbilder postuliert wurde. Allerdings kamen auch bei der Erforschung der europäischen Antike zunehmend andere Kulturen: die Etrusker, die Gallier, Germanen usw. ins Blickfeld. Deren teils sehr einfache oder formal anders gestaltete Götterbilder traten nun neben die ideal-schönen antiken Statuen und erzeugten ebenfalls eine auffallende ästhetische Spannung.

Die Götzenbilder veränderten die Seh- und Darstellungsgewohnheiten zunächst auf einer 'dokumentarischen Ebene'. Sie waren so fremdartig und scheinen sich eingeübten Wahrnehmungs- und Beschreibungsmustern so weitgehend entzogen zu haben, dass für sie neuartige Modi der reproduktionsgraphischen Wiedergabe benutzt werden mussten: Nachdem besonders ungewöhnliche oder formal anspruchsvolle antike und moderne Statuen schon seit den 1520er Jahren in zwei Ansichten, von vorne und hinten, im Druck publiziert worden waren, wurde nun etwa in Cartaris und Pignorias mythologischem Handbuch von 1624 ein „idolo d'avorio“ systematisch in gleich vier Holzschnitten erfasst (Abb. 3a-d). Und mit noch einer weiteren Neuerung wartete diese Publikation auf: In ihr wurde der 'ethnographische[...] Bildvergleich', den González de Mendoza nur in der Beschreibung entwickelt hatte, erstmals auch in Bildtafeln vor Augen geführt, etwa indem Bildwerke aus Amerika mit ägyptischen Idolen in Bezug gesetzt wurden (Abb. 4). ${ }^{28}$ Bei zweidimensionalen Bildern bemühte man sich um stilistisch möglichst getreue 'Faksimilie'-Reproduktionen. In Samuel Purchas Reisebuch von 1625 sind so fast alle Seiten des Codex Mendoza (entstanden 1541/1542; heute Bodleian Library, Oxford) zur aztekischen Religion, Kultur und Geschichte wiedergegeben. Athanasius Kircher in seiner China illustrata (1667) und wohl in dessen Gefolge Olfert Dapper (1672) bemühte sich dann etwa, den Stil chinesischer Holzschnitte

26 Die bisherige Forschung zu den Anfängen dieser Fragen verlegen diese häufig erst ins zweite Viertel des 18. Jahrhunderts, vgl. etwa Mitter 1977 und Connelly 1995.

27 Zur Geschichte siehe Buddensieg 1965; Cole 2009.

28 Seznec 1931; Lein 2002; Cummins 2010; Effinger/Logemann/Pfisterer 2012, $17-20,170-173$ (Kat. II.14). 
möglichst exakt kopieren zu lassen (Abb. 5). ${ }^{29}$ Dies sagt zwar noch nichts über die ästhetische Wertschätzung der Objekte aus. Aber dem eingehenden dokumentarischen Interesse an deren Gestalt lassen sich bereits früh Aussagen zur Seite stellen, die explizit die Kunstfertigkeit der fremden Völker in Afrika, Asien und Amerika rühmen. Albrecht Dürers etwas unspezifisches Lob der „ingenia“ dieser Künstler wird ergänzt durch Aussagen etwa von Duarte Pacheco Pereira (1505-1508) und Valentim Fernandes (1506-1510) über die Schnitzer in Sierra Leone, die „die schönsten Elfenbein-Löffel“ herstellten bzw. „sehr begabt [...] in der manuellen Herstellung [...] von Gegenständen aus Elfenbein“ "seien, oder aber durch das Lob afrikanischer Webereien. ${ }^{30}$ Die Kunstfertigkeit der nordamerikanischen Indigenen diente schon Bartolomé de las Casas als Argument für deren entwickeltes Menschentum; Theodor de Bry schwärmte dann 1590 in der Einleitung seiner America-Serie von der Tugend, Geistesschärfe und Geschicklichkeit in der Neuen Welt:

Dann wiewol sie Gottes vnd seines heyligen Worts keine Erkan[n]tnusz haben / auch gar nichts studiert/so vbertreffen sie dannoch vns in vielen stücken/ als in mässigkeit desz Lebens/vnd scharpffsinnigkeit desz Verstandes/daher sie wissen so hübsche vnd schöne Ding/ohn einig eysern oder ander Instrument/zu zurichten/ dasz es vns vngläublich zu seyn bedüncken würde/wann es nicht die Engelländer selbst in ihren Reysen/so sie in deren Landschafft gethan/versucht hetten. ${ }^{\text {" }} 1$

Bei allen diesen Beispielen mag es vorrangig noch um kunsthandwerklichtechnische Aspekte gehen-wie im übrigen zumeist auch bei den Erwähnungen südamerikanischer Federbilder. ${ }^{32}$ Zumindest die „kunstreiche[n] Goldtarbeiter" in Peru produzierten aber offenbar trotz einfacher Werkzeuge ästhetisch herausragende Gegenstände und Bildwerke aus Gold und Silber, ja sie ahmten die gesamte Natur in Metall nach. Und dies alles

29 Dazu Honour 1976, 165 f. und Effinger/Logemann/Pfisterer 2012.

$30 \mathrm{Zu}$ den Schnitzereien siehe Bassani 2000, 25, 28; zu afrikanischen Geweben aus Raffiabastfasern etwa Cavizzi 1694, 207: „Ja etliche deren scheinen zu seyn ein weicher und linder Sammet/wie nit weniger als hoch und lebhafft an der Farb/ dass sie die unsrige nit selten übertreffen."

31. Theodor de Bry, unpaginierte Vorrede zu Harriot/Bry 1590. Zu las Casas' Argumentation Pfisterer 2011; allerdings gab es auch deutliche Negativurteile, vgl. nur MacCormack 2006.

$32 \mathrm{Zu}$ dieser Technik zusammenfassend Russo/Wolf/Fane 2009.-Vgl. aber etwa Tarde 1984, 41 zu den Exemplaren 1593 im „Cabinet du Pape“: "quelques tableaux bien peints de peinture faite de petites plumes de diverses couleurs si bien rapportées et si bien jointes et collées que qui n'y advise de bien près, la prend pour une peinture ordinaire faite de bonne main, [...]." 
585 Parte Seconda

S.

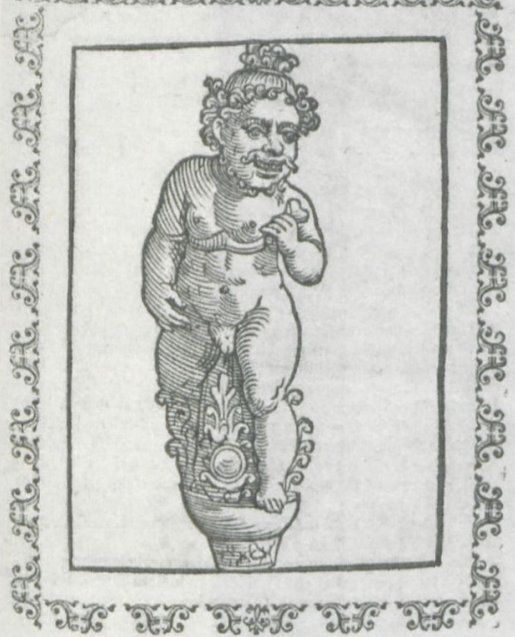

\section{Seconda Parto :}

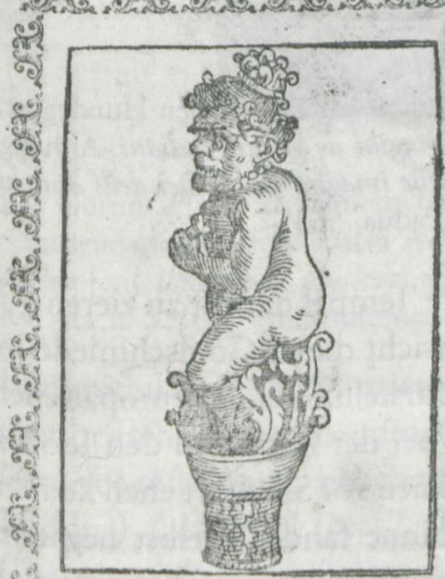

Ses.

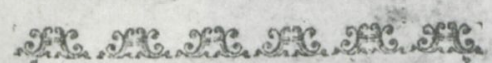

Delle Imagini de i Dei. 587

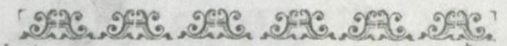

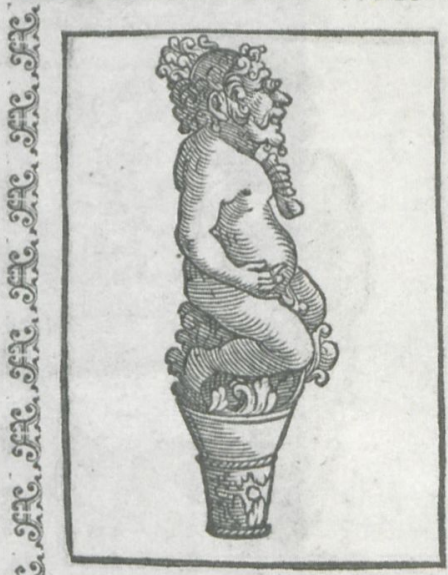
है I3.

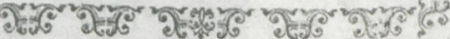

Delle Imagini de i Dei. 587

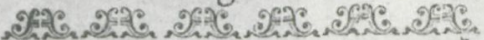

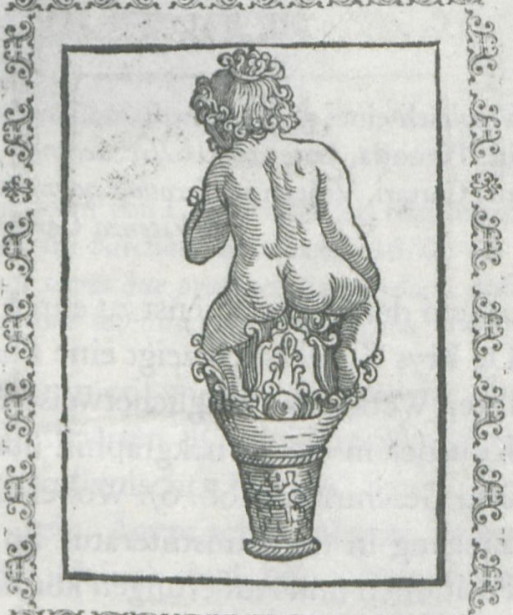

")

$$
\text { I L. F I N E. }
$$

Abb. 3 a-d

Vier Ansichten eines „idolo d'avorio“, in: Pignoria, Lorenzo (1626): Seconda parte delle imagini de gli dei indiani. Anhang zu: Cartari, Vincenzo: Seconda novissima editione delle imagini de gli Dei delli antichi di Vicenzo Cartari Reggiano. Padua, 586 f. 


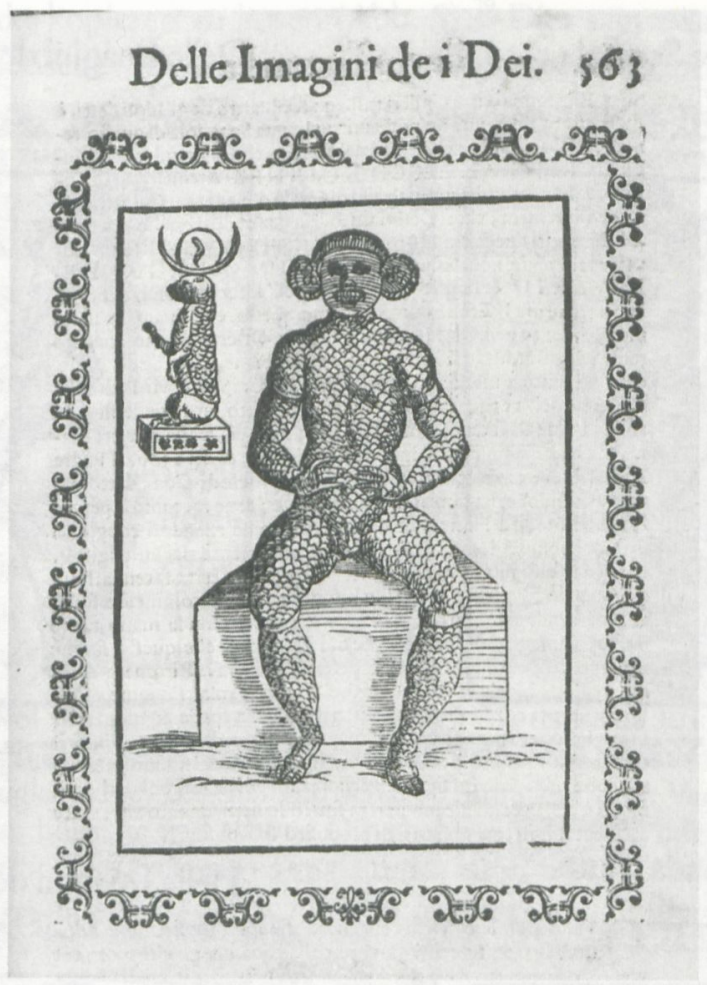

Abb. 4

Vergleich eines geschuppten amerikanischen Idols mit einem ägyptischen Hundegott, in: Pignoria, Lorenzo (1626): Seconda parte delle imagini de gli dei indiani. Anhang zu: Cartari, Vincenzo: Seconda novissima editione delle imagini de gli Dei delli antichi di Vicenzo Cartari Reggiano. Padua, 563.

„allein dem Gottesdienst zu ehren/ unnd ihre Tempel darmit zu zieren. “33 De Brys Kupferstich zeigt eine Phantasie-Ansicht dieser Goldschmiede in ihrer Werkstatt-möglicherweise die erste Darstellung außereuropäischer Künstler in der Druckgraphik überhaupt - bei der Arbeit an den Idolen und Geschirren (Abb. 6). Wobei diese Positionen vor Sandrart eben keinen Eingang in die Kunstliteratur im engeren Sinne fanden. Selbst negative Positionen und Äußerungen können letztlich als Indizien dafür verstanden werden, dass nun das Verhältnis von europäischer zu außereuropäischer

33 Benzoni/Höniger/Bry 1597, Taf. XXVII.-Vgl. etwa schon Cortez 1986, $100 \mathrm{f}$. an Karl V., wo angesichts der Werke aus Gold und Silber neben deren „Neuheit“ und "Seltenheit" auch die kunsthandwerkliche oder künstlerische Qualität gerühmt wird. 


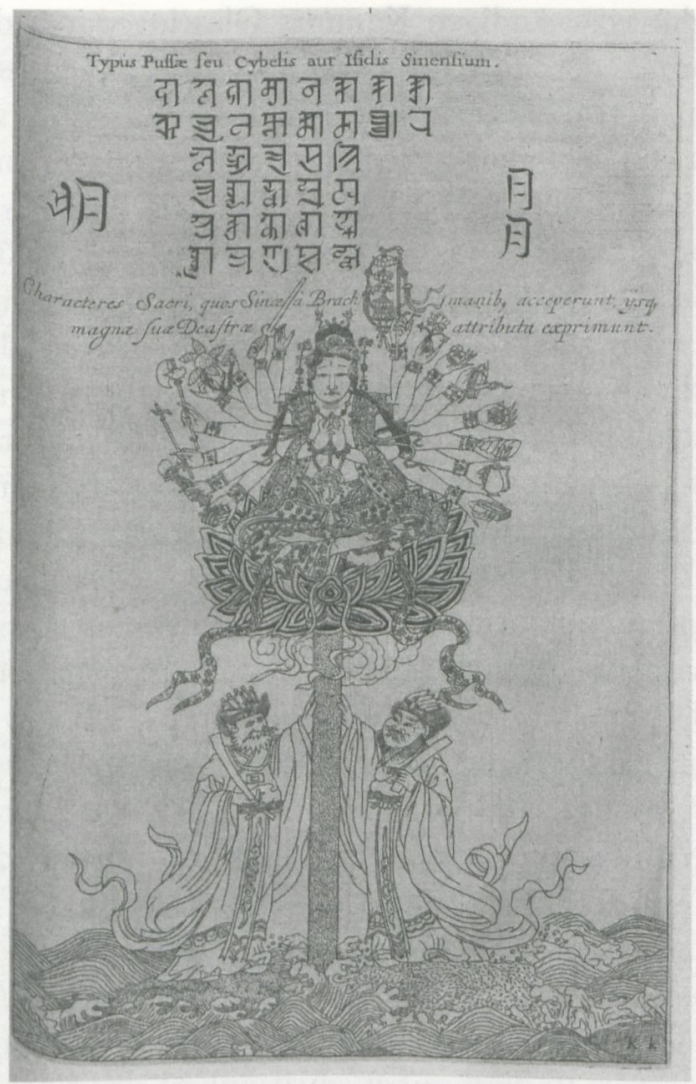

Abb. 5

Die Göttin „Pussa“ als chinesische Erscheinungsform von Cybele oder Isis (eigentlich Tausendarmiger Bodhisattva Avalokiteshvara), in: Kircher, Athanasius (1670): La Chine [...] illustrée de plusieurs monuments tant sacrés que profanes. Amsterdam, Taf. Kk [nach S. 190] [Illustrationen entsprechen der lateinischen Erstausgabe 1667].

Bildproduktion differenzierter wahrgenommen wurde. So war in der Gottorfischen Kunstkammer, deren illustrierten Katalog 1666 Adam Olearius publizierte, nebeneinander ein „Indianischer Pagode“ (Nr. 1, ein Buddha), „Horus“ (Nr. 2, Uschebti), „ein Ägyptischer Abgott voller Characteren“ in zwei Ansichten (Nr. 3,4 Osiris) sowie ein „Abgott der Nordländer“ zu sehen, dahinter aber eine Ikone des Heiligen Nikolaus"Muskowitisch Bild/S. Nicolai“ besagt die Erläuterung zur Tafel (Abb. 7) ${ }^{34}$ Aus der protestantischen Sicht des Schleswiger Fürsten und des

34 Spielmann/Drees 1997; Stephanie-Gerrit Bruer (2010), in: Kunze, $60 \mathrm{f}$. (Kat. 2.8). 
für die Sammlung zuständigen Kurators Olearius waren dies alles gleichermaßen Formen der Idolatrie, wobei das altgläubig-christliche Europa, für dessen Extremform offenbar die Orthodoxen Russlands stehen, nun in einen Kontext mit den nordischen Rändern Europas, dem Außereuropäischen und der Antike rücken konnte. Dies war keine Ausnahme: Samuel van Hoogstraten verdammt in seinem Malereitraktat von 1678 ähnlich die „Taschenspielertricks" illusionistischer Malerei in ägyptischen Heiligtümern, wo die Götzen so platziert gewesen seien, dass die aufgehende Sonne ihre Kronen in Brand zu setzen schien-Verfahren, wie es sie laut Hoogstraten ähnlich immer noch in katholischen Kirchen in Fortsetzung dieser Abgötterei gebe. ${ }^{35}$ Dagegen vertrat Nehemiah Grew in seinem nicht realisierten Katalog-Konzept für die Sammlung der Royal Society die Auffassung, europäische und außereuropäische (ethnologische) Objekte seinen unter gemeinsamen Kategorien auszustellen. ${ }^{36}$

Explizit wird die Relativität von Schönheitsvorstellungen seit dem späteren 17. Jahrhundert ebenfalls thematisiert. Dabei wird schon im Zusammenhang mit der Querelle des Anciens et des Modernes - allen voran von Charles Perrault und Abbé DuBos-zwischen einem beau absolu und einem beau relatif, einer Schönheit abhängig vom historischen und geographischen Kontext (den jeweiligen mours et usages also, für die die Antike nicht immer gleichermaßen Maßstab sein kann), unterschieden, wobei letzeres Konzept zunehmend an Bedeutung gewann (außereuropäische Handwerks- und Kunstprodukte spielten dabei als Argument freilich noch eine untergeordnete Rolle). ${ }^{37}$ Aber auch außerhalb dieses Diskussionskontexts finden sich relevante Überlegungen, so wenn Gérard Audran 1683 in seinem populären Lehrbuch zu den idealen menschlichen Proportionen vorschlägt, als Ausweg aus dem Streit der Künstler um unterschiedliche Schönheitsvorstellungen die Antike zum Vorbild zu nehmen. Ort, Klima und Menschentyp hätten damals besonders wohlgestaltete Menschen hervorgebracht. Wobei die überragende Schönheit antiker Götterstatuen aber eben auch daraus resultieren würde, dass die Künstler in sie ihre ganze Devotion gelegt hätten und ihr Bildschaffen zu einer Art Gottesdienst geworden sei, wodurch sich auch auf Seiten der Betrachter die

35 Vgl. Hoogstraten 1678, 211.

36 Vgl. Collet 2007, 336 f., den allerdings der Umstand, dass der Entwurf nicht realisiert wurde, zu einer negativen Beurteilung der Wertschätzung fremder Objekte noch im späten 17. Jahrhundert führt.

37 Dazu Jauß 1964; Gisi 2007; zur weiteren Relevanz dieser Unterscheidung Zelle 1995. 


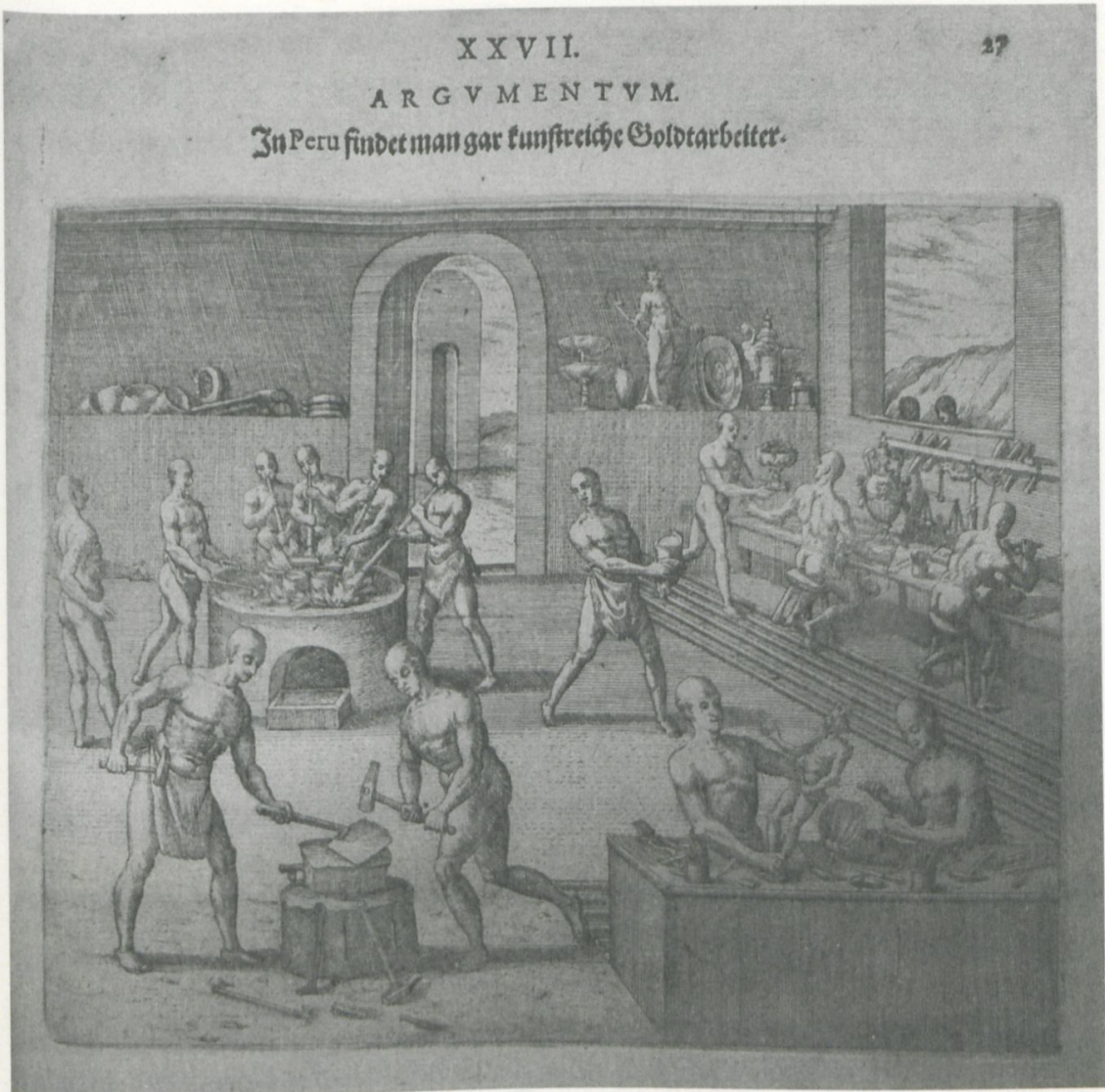

Abb. 6

Goldschmiede in Peru, in: Benzoni, Girolamo (1597): Neuwe und gründtliche Historien von dem nidergängischen Indien. Hrsg. von Theodor de Bry.

Frankfurt a.M., Taf. XXVII.

Verehrung für die so produzierten, schönen Götter intensiviert hätte. ${ }^{38}$ Diese Gedanken wurden schon früher angedeutet, teils sogar in radikalerer Zuspitzung - so zu Beginn des 17. Jahrhunderts von Christoph Besold in De natura populorum:

38 Audran [1683] 1690, Vorrede. 


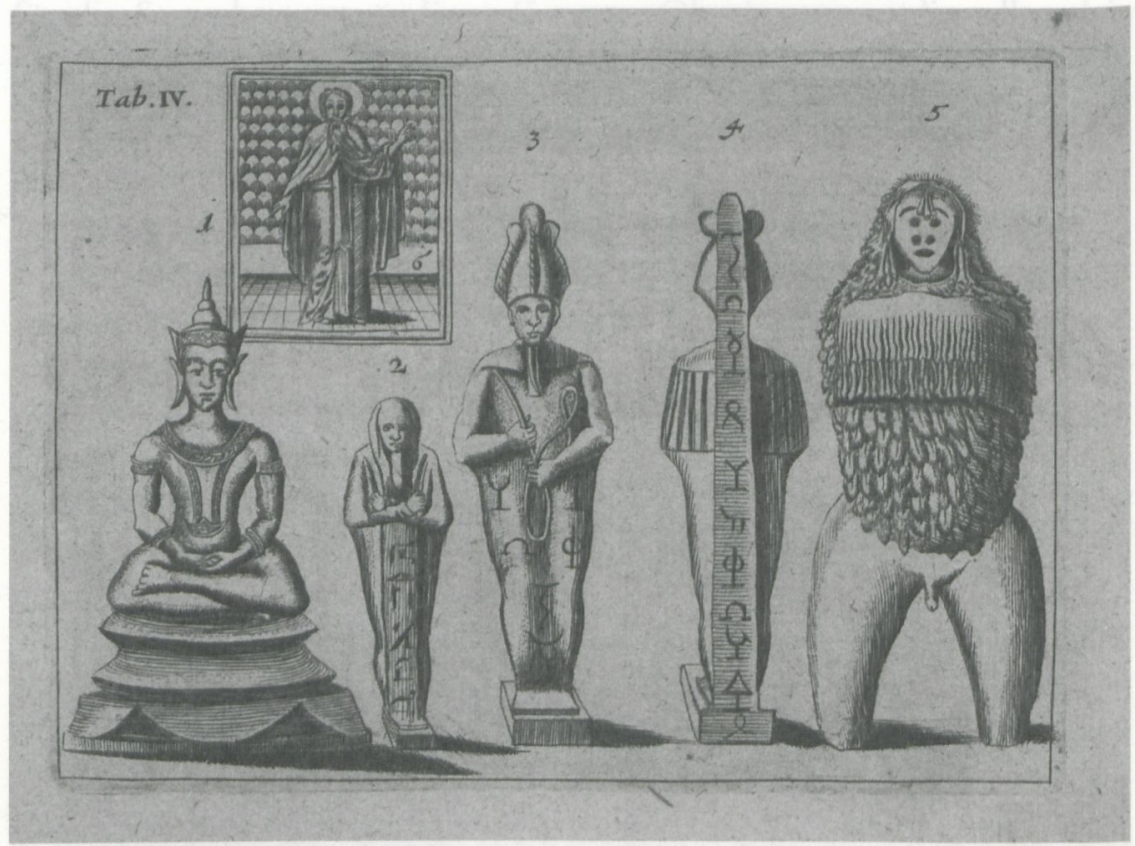

Abb. 7

"Seynd lauter Abgötter", in Olearius, Adam (1674): Gottorffische Kunst-Kammer. Schleswig, Taf. 4.

Zudem ist die Natur der Völker so sehr verschieden und abweichend, daß sie nicht nach der Wohlgefälligkeit oder der Schönheit des Körpers zu urteilen gleichförmig erkannt werden können. ${ }^{39}$

Noch ausführlicher hatte bereits einige Jahrzehnte zuvor Montaigne die Vorlieben der Peruaner für große Ohren, der Mexikaner für schwarze Zähne oder die unterschiedlich favorisierten weiblichen Brustformen in Europa beschrieben, um angesichts dieser pluralen Vorstellungen und Geschmacksrichtungen - und im übrigen auch mit explizitem Verweis auf die eingangs zitierte Passage des Xenophanes zur Anthropozentrik der Gottesvorstellungen-auf die Relativität menschlicher Phantasien, Kategorien und eben auch Schönheitsideale hinzuweisen. ${ }^{40}$ Als spektakulärer Einzelfall sei auch darauf hingewiesen, dass bereits im späten 13. Jahr-

39 Besold 1619, 5: „Ac adeo etiam varia \& discrepans est natura populorum, ut nec de gratia seu pulchritudine corporis, pariliter judicare deprehendantur." - Zitiert (auch die Übersetzung) nach Neuber 1995, 177.

40 So Montaigne in Essais II, 12; vgl. Maspoli Genetelli, 130-141. 


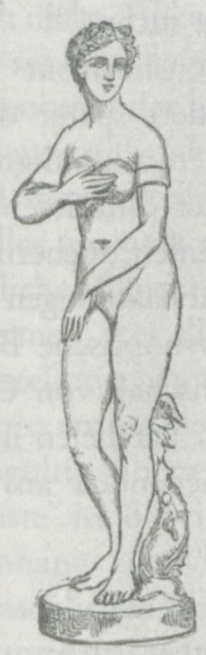

1

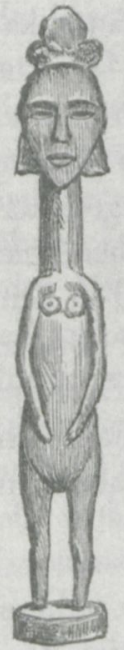

2

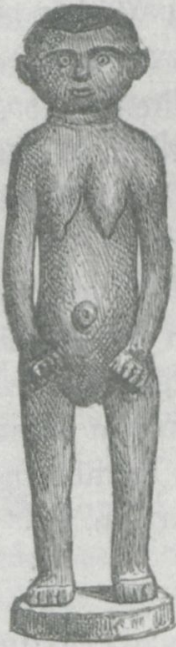

3

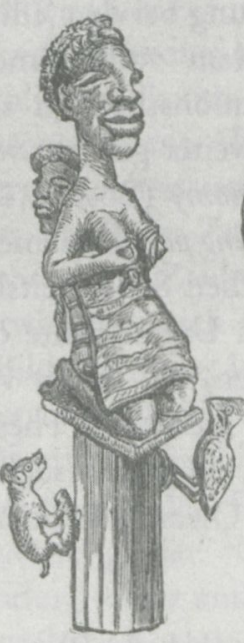

4

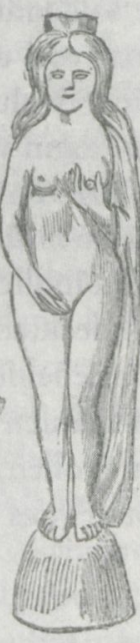

b

Fig, 178.

1. La Vénus de Médicis. - 9, 3. \$. Idoles de l'Afrique occidentale. - 5. Vénus gauloise.

Abb. 8

Die Venus Medici, eine gallische Venus und „westafrikanische Idole“, in: Cleuzion, Henri de (1887): La Création de l'homme et les premiers âges de l'humanité.

Paris, 271, fig. 178.

hundert Witelo die Vielzahl europäischer Körper- und Schönheitsideale benennt. ${ }^{41}$

Komplementär zu dieser vorsichtigen Lockerung des europäischen Schönheitskanons lässt sich verstehen, dass auch die fremden Körper der Nicht-Europäer nun in Kategorien des europäischen Tugend- und Kunstideals beschrieben werden konnten - und teils sogar als weniger dekadent und überlegen den Körpern der europäischen Landsleute. So evozieren Körper und Gesichtsausdruck des Indianer-Helden Canassatego in John Shebbeares Novelle Lydia (1753) die 'schönsten Antiken Roms', den Apollo Belvedere, den Laokoon und den Borghesischen Fechter, und wenig später wird auf Benjamin Wests Gemälde Abschied eines Indianers von seiner Familie der Protagonist ebenfalls die Haltung des Apollo Bel-

41 Witelo: Perspectiva, 4, 148; hrsg. in Baeumker 1908, 175. 
vedere einnehmen - zumindest auf dieser ästhetischen Ebene scheint die antike Vollendung bei den 'edlen Wilden' in Amerika zu suchen. ${ }^{42}$

Erst vor dem vollkommen veränderten Diskussionshorizont von Darwins Evolutionstheorien sollten über ein Jahrhundert später diese Ansätze dann weiter gedacht werden. William Rimmer etwa kündigte in seiner Art Anatomy (Boston $1877 /^{2} 1884$ ) angesichts der differierenden Körper im melting pot Nordamerika den in solchen Zeichenlehrbüchern bis dato verbindlichen Schönheitskanon auf. Und in den Abhandlungen zur neu entdeckten Urgeschichte der Menschheit können synoptische Bildtafeln erscheinen, die in der vergleichenden Zusammenschau von Göttinnen-Statuen die neuen Thesen von ethnographischen Parallelen illustrieren wollen, in der visuelle Anmutung aber immer noch an die „Abgötter" des Olearius erinnert (Abb. 8). ${ }^{43}$

\section{Eine globale Phantasie-Geschichte der Bildkünste}

Der Blick auf die Götterbilder der Welt führte nicht nur dazu, dass deren fremde Formen in neuartiger Weise dokumentiert und vergleichend zusammengestellt wurden; und er trug nicht nur zu der Einsicht bei, dass Götterbilder Ausdruck menschlicher Phantasien und damit relativer ästhetischer Wahrnehmungen und Werte sind. Der Blick auf die Götterbilder der Welt und die damit verbundenen Überlegungen zu historisch und geographisch unterschiedlichen menschlichen Phantasien eröffneten auch eine neue Möglichkeit, die Geschichte der Bildkünste zu konzipieren. Diese war bislang primär (und sollte es auch noch lange Zeit bleiben) eine Fortschritts-Geschichte der immer besseren Naturnachahmung und des Strebens nach einer vermeintlich normativ fassbaren, absolut-idealen Schönheit gewesen, beginnend bei den Ägyptern oder dem noch ferneren, mythischen Orient, dann vervollkommnet in der griechisch-römischen Antike. Darauf folgte eine Zeit des Verfalls in der Spätantike und im Mittelalter, um dann mit der Renaissance einen neuen Höhepunkt zu erreichen, den das 17. Jahrhundert fortsetzte.

Die Überlegungen zu den Götterbildern und die damit verbundenen Andeutungen zu einer Geschichte der Bildkünste fassten das Thema da-

42 Honour 1976, 125; vgl. 120 die Hinweise, dass die 'Indianer' bereits im 17. Jahrhundert mit Spartanern oder den alten Römern verglichen wurden; dazu auch Kohl 1986 und Lupher 2003.-Zu Wests Gemälde Röttgen 1998.

43 Dazu Bostwick Davis 2002; Pfisterer 2009. 
gegen chronologisch und geographisch viel weiter. Vor allem setzten sie auch nicht erst auf der Ebene konkreter künstlerischer Formentwicklungen an, sondern basierten auf einer Analyse von grundlegenden Entwicklungsstufen der bildproduzierenden menschlichen Phantasie. Vier Phasen schienen sich dabei abzuzeichnen: Die Menschen Asiens, Afrikas, Amerikas und offenbar teils auch der europäischen Vergangenheit verehrten zunächst bildlos ein oder mehrere höhere Wesen. Im Laufe der Zeit konnten persönliche Objekte der Verehrung, 'Fetische' und Zauberobjekte dazu kommen. Aus diesen Einzelphantasien entwickelten sich dann kollektive Vorstellungen von Idolen und Rituale der Bildverehrung. In einem vierten Schritt wurde diese Abhängigkeit der Gottesvorstellungen von Bildwerken endgültig überwunden. Diese 'globale Phantasie-Geschichte der Bildkünste' ist dabei teils wohl als Vorlauf, teils auf jeden Fall im Zusammenhang und komplementär zur Erforschung der 'Geschichte des menschlichen Verstandes' und der Begründung einer anthropologischen Historie im 'langen' 18. Jahrhundert zu verstehen: Auch hierbei spielte die Analyse zeitlicher und geographischer Ausprägungen der menschlichen Phantasie und verschiedener Mythologien und Göttergestalten als ihrem maßgeblichen Ausdruck eine zentrale Rolle. ${ }^{44}$

Einschränkend muss allerdings gleich an dieser Stelle gesagt werden, dass es offenbar keine umfassend ausformulierte zeitgenössische Theorie und Systematik der hier rekonstruierten Überlegungen gab, vielmehr erschließt sich das postulierte Gesamtbild erst aus der nachträglichen $\mathrm{Zu}$ sammenschau verstreuter Bemerkungen. Dazu kommt, dass auch die partiellen Einteilungen und Begründungen für diese Prozesse im Laufe des 17. und 18. Jahrhunderts beträchtlich variieren konnten. Die unterschiedlichen, teils radikal widersprüchlichen Positionen-von etablierten Dekadenz- bis hin zu neuen Polytheismus-Theorien - müssten dafür ausführlich und detailliert dargelegt werden. ${ }^{45}$ Hier können freilich nur einige Text- und Bildbelege die These von der Vorstellung der vier PhantasieStufen etwas genauer beschreiben.

Die früheste Phase der Menschheitsgeschichte, häufig bis zur Sintflut datiert, und ihre Verehrung göttlicher Kräfte war nach Vorstellung der Frühen Neuzeit bildlos. Diesen Zustand glaubte Kolumbus bei seiner Ankunft in Amerika offenbar vorgefunden zu haben, wenn er 1493 von den Ureinwohnern behauptet, sie würden keine institutionalisierte Religion kennen noch seien sie Götzendiener, sie glaubten vielmehr, dass alle

44 Dazu umfassend Gisi 2007.

45 Vgl. etwa Schmidt 1985; Miller 2001; Mulsow 2001; Häfner 2003; Mulsow 2005. 
Macht und alles Gute vom Himmel käme, weshalb sie die Natur und allem voran die Sonne verehrten. ${ }^{46}$

Die zweite Stufe wurde erreicht, als die Menschen aufgrund ihrer individuellen, mehr oder weniger zufälligen Phantasien und Vorstellungen bestimmte Gegenstände für die Verehrung herauszuheben begannen. Dieser Zustand schien sich für das 17. und 18. Jahrhundert besonders gut in Afrika und dem dortigen Glauben an Fetische beobachten zu lassen: (Persönliche) 'Fetische' - den Begriff fetisso in dieser Bedeutung prägten die Portugiesen an der afrikanischen Künste zu Beginn des 16. Jahrhunderts-sind Dinge, die jeder für sich aufgrund seiner eigenen Phantasie erwählt. ${ }^{47}$ Godefroy Loyer formuliert diesen Standpunkt zu Beginn des 18. Jahrhunderts wohl in größter Prägnanz: „Ces fétiches sont diverses, selon la diverse fantaisie d'un chacun", oder auch: "Chacun fait lui-même ses fetiches, et les choisit à sa volonté. “ ${ }^{48}$ In einem von Theodor de Brys Bänden der India Orientalis-Reihe zum Königreich Guinea zeigt ein Kupferstich nicht nur diesen Fetisch-Glauben und Zauberpraktiken, sondern im Hintergrund auch einige Riten wie das Einhalten eines wöchentlichen Feiertages, die angeblich als Relikte der vorsintflutlichen Urreligion zu deuten sind und auch in dieser zweiten Stufe fortbestehen (Abb. 9). Charles de Brosses wird dann 1760 die erste buchlange Abhandlung zu diesen Fetisch-Göttern schreiben, sie mit frühen Stufen der ägyptischen Religion vergleichen und vor allem auch ihre kontingente Entstehung betonen im Unterschied zu einer Reihe von vorausgegangenen Theorien, etwa von Thomas Hobbes, David Hume oder Giambattista Vico, die die Entstehung von Religionen aus der Furcht der Menschen erklären wollten. ${ }^{49}$

Im bis zu Konfuzius ebenfalls götterbildlosen China begannen sich dagegen - so Athanasius Kircher und ander ${ }^{50}$ - nicht aus eigenem Antrieb,

46 Vgl. Jane 1960, 196; dagegen schienen Amerigo Vespucci die 'Wilden' in Südamerika, die wie die Epikuräer ohne Glaube an Götter und Religion lebten, noch schlimmer als die Heiden; Vespucci 1992, 50, 64.-Zu Vorstellungen historischer Stufen der Idolverehrung in Amerika siehe MacCormack 2006.

47 Dazu grundlegend Pietz 1983, 1987 und 1988.

48 Loyer $1714,213,217$.

49 Zit. nach der deutschen Übersetzung: Über den Dienst der Fetischen Götter oder Vergleichung der alten Religion Egyptens mit der heutigen Religion Nigritiens. Berlin u. a. 1785 .

50 Cousin 1688, 13-18 „Avertissement“ behauptet, die Chinesen „depuis le commencement de leur Origine, jusque au tems de Confucius, n'ont point été Idolâtres, qu'ils n'ont eu ni faux Dieux, ni statues, qu'ils n'ont adoré que le Créateur de l'Univers, qu'ils ont toûjours appellé Xam-ti, \& auquell leur troisième Empereur, nommé Hoam-ti, bâtit un Temple, qui apparemment a été le premier qu'on ait bâti 


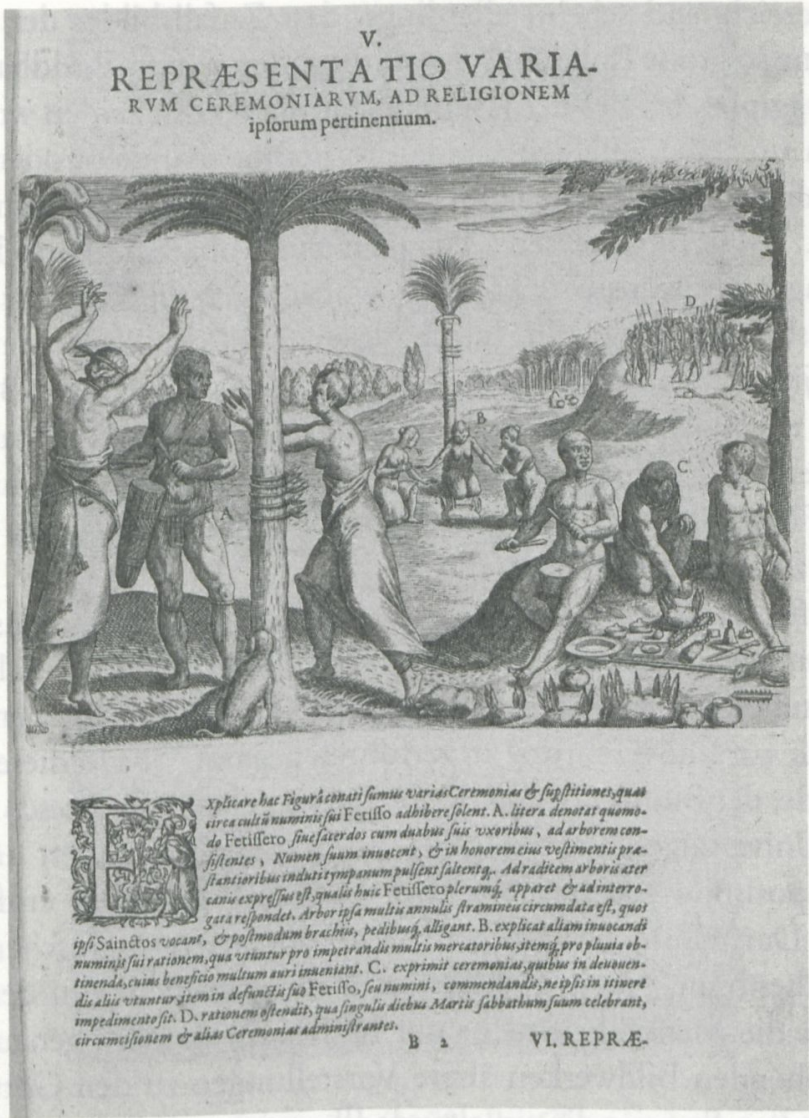

Abb. 9

Afrikanischer Fetisch-Glaube, in: Bry, Theodor de/Bry, Johann Israel de (Hrsg.) (1604): Pars VI Indiae Orientalis. Vera Et Historica Descriptio Avriferi Regni Gvineae. Frankfurt a.M., Taf. 5.

sondern unter dem Einfluss der Idolatrie Indiens, die ihrerseits wiederum von den Ägyptern abstammte, die Phantasie kollektiv auf Götzenbilder auszurichten. In China wird durch diese Konstellation sozusagen die zweite Stufe fast ganz übersprungen. Kircher äußert sich in seiner China illustrata 1667 zwar nicht weiter zur Entwicklungsgeschichte der dortigen Bild-

à Dieu. Le nom de Xam-ti, qu'ils donnoient à Dieu, signifie, Souverain Maître, ou Empereur. [...] Mais enfin [nach Konfuzius], lors qu'on eut apporte' des Indes l'Idole de Foe [den Buddha], c'est-à-dire, soixante-cinq ans aprez Jesus-Christ, ce torrent [Aberglaube und Götzendienst] se deborda si fort, qu'il fît un ravage, dont les tristes effets se voyent encore aujourd'hui."-Dazu Mungello 1987. 
künste. Bezeichnend scheint allerdings, dass Zufallsbilder der Natur für Kircher eine so große Rolle spielen - unter anderem eine Buddha-Figur auf einem Berggipfel, bei der der Jesuitenpater nicht entscheiden wollte, ob es sich allein um eine zufällige Gesteinsformation handelte oder eine von Menschenhand im 'natürlichen' Bergmassiv realisierte Monumentalfigur (Abb. 10). ${ }^{51}$ Man muss diesen Gedanken an europäische Vorstellungen zu den Anfängen der Künste rückbinden, wonach sich die Skulptur insgesamt aus Zufallsbildern der Natur, in die die menschliche Phantasie Gestalten hineinprojizierte, entwickelt habe. So verstanden erscheint der Buddha auf dem Berg ebenfalls als Anfangsfigur, bei der die projektive Phantasie mit der Zeit ein allgemein anerkanntes Götzenbild geschaffen hat. Dieses „Idolum FE in Monte expressum“ würde für China daher den Übergang von Stufe zwei zu drei markieren.

Auch für Europa und den Orient ließ sich in Zusammenschau von Textquellen und monumentaler Überlieferung eine götterbildlose Frühphase postulieren-bevor die Wirkung der ersten (griechischen) Statuen langsam alle zur Bildverehrung zu verführen begann. ${ }^{52}$ Es ist dieser „Anfang der Idolatrie, der Bildwerke und der Buchstaben “, den Francesco Bianchini in seiner Universalgeschichte (1697) ins 19. Säkulum datiert und mit einem Kompositbild aus verschiedenen antiken Bildwerken eröffnet, darunter eine Darstellung des Prometheus als des ersten mythischen Bildners der Menschenfigur. Noch wichtiger aber ist, dass Bianchini der Idee anhängt, dass die Menschheit nicht nur in Texten, sondern genauso in den nun entstehenden Bildwerken ältere Vorstellungen zu den Göttern quasi konserviert hat. So zeigt die einleitende Illustration zur ersten Epoche und dem Ursprung der Welt verschiedene Symbolbilder zu Chaos und Ordnung der Welt-Zodiak und Circus der griechisch-römischen Antike genauso wie ein Rentier aus De Brys America-Werk-, deren angebliche Vergleichbarkeit Bianchini als Beweis dafür nimmt, dass sich hier ein gemeinsames Wissen von den Anfängen und den Versuchen der 'Bannung' im Bild erhalten habe und wieder rekonstruieren lasse. ${ }^{53}$

51 Kircher 1667, 161 - 173, vgl. etwa auch 205; zur Deutung Chang 2003 und Odell 2009.

52 Varros Bericht dazu hatte Augustinus in De civitate Dei 4, 31 überliefert, fast wörtlich aufgegriffen etwa noch bei Baudelot de Dairval 1686, Bd. 1, 90-95: „les Romains ont été 170 ans, sans avoir ni de statuës, ni de Peintures; ce que les Allemans, les Perses, les Sythes, \& les Lacedemoniens ont observé de même pendant long-temps."

53 Vgl. Sölch 2007, 42-54. 


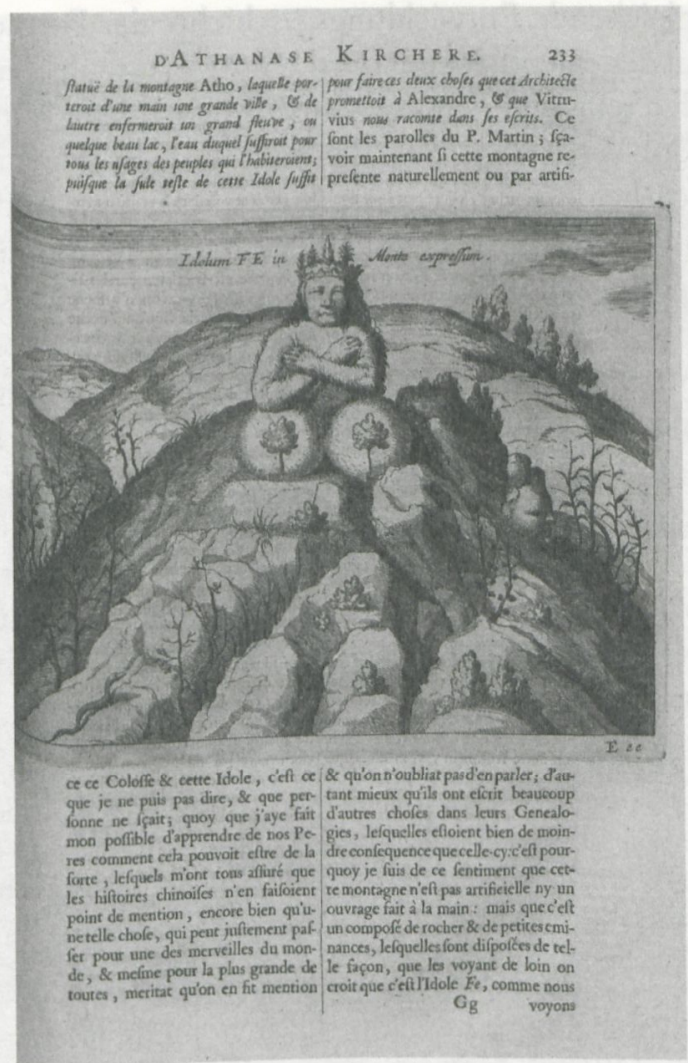

Abb. 10

„Idolum FE“, in: Kircher, Athanasius (1670): La Chine [...] illustrée de plusieurs monuments tant sacrés que profanes. Amsterdam, 233,

[Die Illustrationen entsprechen der lateinischen Erstausgabe 1667].

Diese mit der Erfindung der Idolatrie und der Bildwerke eröffnete dritte Phase umfasst dann die künstlich hergestellten und geformten Götterbilder, die als Resultat der kollektiven, kulturell vereinheitlichten Phantasie idolatrischer Völker erscheinen. Der gestochene Titel zu Gerard Vossius' Theologia Gentilium (1668) und eine Tafel aus Joseph-François Lafitaus Mours des Sauvages (1724) stellen die Genese und Entwicklungsschritte der Idole von der Verehrung von Naturobjekten bis hin zu elaborierten Götterstatuen dar (Abb. 11, 12). ${ }^{54}$ Auch wenn diese Tafeln in religionsgeschichtlichen und ethnologischen Kontexten zu finden sind: Sie lassen sich auch ohne Text auf visueller Ebene rezipieren und präsentieren

$54 \mathrm{Zu}$ diesen Autoren Häfner 2003 und Mulsow 2012. 
dann eine vergleichende Entwicklungsgeschichte der Formen und Skulpturen, wie sie in der eigentlichen Kunstliteratur erst in den Jahrzehnten um 1800 anzutreffen sein wird. Dabei zählte für reformierte Autoren zu diesen Formen der Idol-Verehrung auch der katholische Bildgebrauch. Antike, außereuropäisch-heidnische wie innereuropäische, aber anders-konfessionelle Vorstellungen erscheinen in diesem Argumentationszusammenhang in einer Kategorie, wie schon an der Präsentation der Gottorffischen Kunstkammer beobachtet. Kaum erwähnt werden muss, dass dagegen die katholischen Autoren die aus ihrer Sicht grundlegende Differenz ihrer auf einen Prototypen ausgerichteten Bilderverehrung zu den das materielle Bildwerk vergöttlichenden Idolatrien herausstellten. Auch dies ließ sich allerdings anders deuten: Thomas Astley etwa erkannte 1746 vollkommen richtig, dass die katholische Kirche Bilder nur als 'Durchgangsmedien der Verehrung' verstand - eine Vorstellung, die er allerdings auch als Rechtfertigung der Fetische von den Einwohnern der Afrikanischen Goldküste gehört haben will:

This is the same Apology [für die Fetische] which the Romish Church and Priests make for their Images, it is plain, [...] that they consider their Fetishes, only as material Objects qualified with certain Virtues and Powers, by the supreme Deity, for the Benefit of his Creatures [d.h. die Fetische selbst wurden eben nicht als Götter angesehen]. ${ }^{55}$

Die vierte und letzte Stufe dann ist wieder die ganz oder weitgehend bildlose Gottesverehrung eines aufgeklärten menschlichen Geistes, der keiner sinnlichen Hilfsmittel für seine Gottesvorstellung bedarf, soweit dies überhaupt möglich ist.

\section{Zusammenfassung}

Zusammenfassend: Die erkenntnistheoretische Einsicht bereits der Antike, dass Götterbilder Projektionen der Phantasie und Produkte der Kunst sind, erfährt durch das zunehmende Bekanntwerden außer-europäischer Götterbilder neue Impulse und Herausforderungen. Gerade die Diskussionen um Idolatrien und Götterbilder weltweit, ein Bereich, wo man das stärkste Alteritäts- und Wertgefälle aus europäischer Sicht vermuten würde, gerade diese Diskussionen um fremde Formen, die zugrunde liegenden schönen Phantasien und die sich in diesem Zusammenhang abzeichnende 'neue Geschichte der Kunst' führten zu Ansätzen einer Relativierung der euro-

55 Astley 1746, 25; auf diese Stelle verweist schon Pietz 1983/1987. 


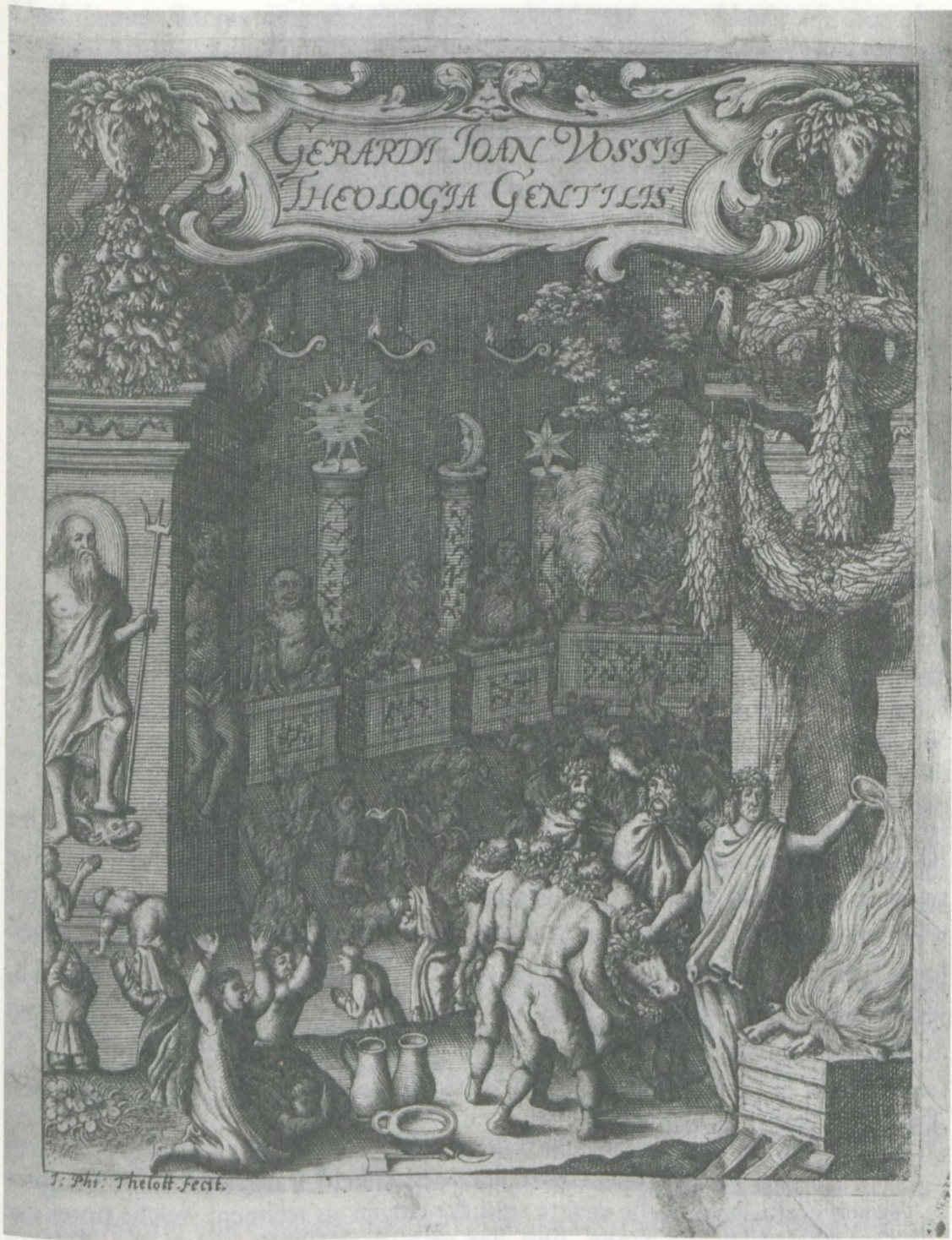

Abb. 11

Entwicklungsstufen der Idol-Verehrung aus Naturformen, in: Vossius, Gerardus J. (1668): De theologia gentili et physiologia christiana. Frankfurt a.M., gestochener Titel.

päischen Wahrnehmung, Einschätzung der künstlerischen Phantasien und der ästhetischen Werte. Diese erstaunlichen Ansätze in Text- und vor allem auch Bildform dokumentieren eine entscheidende und eben auch ästhe- 


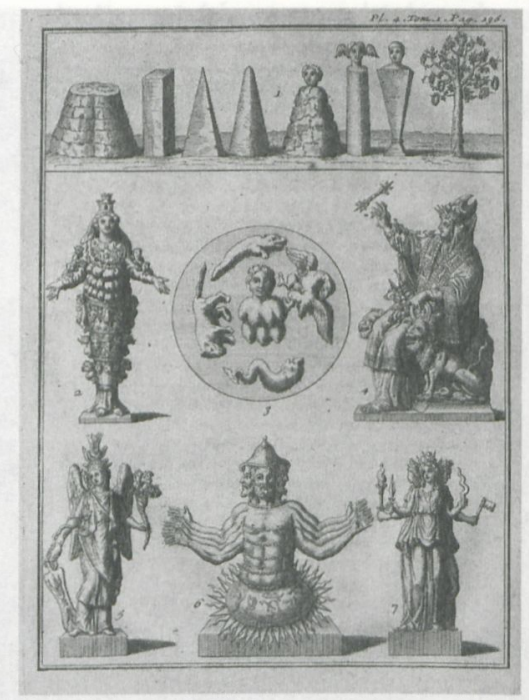

Abb. 12

Entwicklungsstufen der Idol-Verehrung aus Naturformen, in:

Lafitau, Joseph-François (1724): Mours Des Sauvages Amériquains, Comparées Aux Moeurus Des Premiers Temps.

Paris, Bd. 1, Taf. 4.

tische Irritation. Dass sich diese nicht gleich vollumfänglich in der Kunstliteratur niederschlug, überrascht wenig - die hier skizzierten Ansätze wurden offenbar erst ab der Mitte des 19. Jahrhunderts konsequenter weitergedacht. ${ }^{56}$ Die „Verschränkung von Einbildungskraft und Mythologie“, von Anthropologie, Ästhetik und Geschichte im Laufe des 18. Jahrhunderts hat Lucas M. Gisi für die anderen (Text-)Disziplinen jüngst ausführlich dargelegt. ${ }^{57}$ In der Geschichtsschreibung jedenfalls sollte 1764 Isaak Iselin ein umfassendes Modell für die geistigen Entwicklungsstufen der Menschheit formulieren:

Wir können sie [die Geschichte der Menschheit] indessen in drey Hauptclassen eintheilen. In die erstere sind diejenigen zu rechnen, welche unter der Oberherrschaft der Sinne, und der sinnlichen Triebe gleich den Thieren stehen, welche die Einbildungskraft sehr wenig, und ihren Verstand gar nicht erhöhet haben. ${ }^{58}$

56 Vgl. dazu etwa Pfisterer 2008; Basu 2012.

57 Gisi 2007.

58 Iselin 1779, 182; dazu Zedelmeier 2003, 246-259. 
Und James Barrys erste Vorlesung an der Londoner Akademie aus den 1780er Jahren unter dem Titel On the History and Progress of the Art lässt immerhin erahnen, wie diese Ideen selbst in solchen Institutionen ankamen:

It is curious to reflect that the exertions of art seem to arise from the disappointment of the human mind, sated, disgusted, and tired with the monotony of real persons and things [...]. In proportion to the serenity and goodness of the mind, it naturally turns way from such a state of things, in search of some other more grateful and consoling; [...]. Hence it arises, that the minds of men in all ages and places where they were at leisure [...] found consolation in the objects of religion, which they would anticipate and realize by their endeavours to cut and carve them in blocks of wood or stone; whether detached from their parent rocks, and set up in high and honoured places of frequent resort, or, as was probably the more ancient way, cut into and making part of immense excavations, as it seems in the mountains of India. Whether this subject matter of religion be well or ill reasoned upon [...], whether it be taken from the various incarnations of the Indian Vistnou, the more elegant forms and ideas of the Greek mythology, or from the more consoling and happily adapted matter which result from the more rational hopes and fears inculcated by the Christian religion; yet the whole together forms an asthonishing chain of the most indubitable proof of the thirst of the mind for a more satisfactory state of things, and of its natural recurrence of the arts of design, as the first, the universal and natural written language, which in furnishing the means of expressing this universal testimony, affords an opportunity of tracing human nature through an immense tract of ages; [...] what should we have known of the ancient nations, their arts and knowledges, were it not for the stimulus which religion afforded to the human exertions? what other motives ever did or could supply its place? ${ }^{\text {(559 }}$

Die Mehrheit der Vertreter der neuen Disziplin Kunstgeschichte scheinen dennoch zunächst im Gefolge Johann Joachim Winckelmanns (gegen den Barry bezeichnenderweise teilweise argumentierte) damit beschäftigt, ihre eigenständige 'Wissenschaftlichkeit' an kanonischen Objekten zu begründen. Zwar hatte Winckelmann auch entgegen lang gehegter Vorstellungen behauptet, die Anfänge der Kunst rührten nicht (allein) aus einem Kulturtransfer von Chaldäa und Ägypten nach Griechenland und Rom her. Vielmehr habe überall ein unabhängiger religiöser Impuls die Menschen dazu veranlasst, Bildwerke zu schaffen. ${ }^{60}$ Aber das Argument war darauf

59 Barry 1809, $370 \mathrm{f}$; dazu auch Haynes 2012, $78 \mathrm{f}$.

60 Vgl. Winckelmann 1776, 4-6; vgl. auch seine Ausführungen zur frühen griechischen Verehrung von 30 Gottheiten in Form von Steinen nach Pausanias 7, 22, 4 in Winckelmanns Anmerkungen zur Geschichte der Kunst des Althertums, Dresden 1767, 29. - Dazu Bruer 2010. 
ausgerichtet, die Eigenständigkeit der griechischen Kunst und der daraus hervorgehenden Tradition zu begründen, den Blick also zu fokussieren, nicht über Europa hinaus zu lenken. Werke und Argumente aus dem topographisch und disziplinär 'falschen'- religionsgeschichtlichen oder ethnologischen - Kontext, zumal, wenn sie eine solche Revision des Sehens, Denkens und Bewertens verlangten wie die fremden Idole, sollten vor diesem Hintergrund für ein knappes Jahrhundert wieder (ästhetisch) fremder erscheinen, als sie es schon einmal gewesen waren. 


\section{Bibliographie}

Astley, Thomas (1746): A New General Collection of Voyages and Travels. Bd. 3. London: Astley.

Audran, Gérard (1690): Des menschlichen Leibes Proportionen [...]. Nürnberg: Sandrart/Froberg [Neudruck der franz. Ausgabe 1683].

Baeumker, Clemens (1908): Witelo, ein Philosoph und Naturforscher des 13. Jahrhunderts. Münster: Aschendorff.

Bailey, Gauvin A. (1999): Art on the Jesuit Missions in Asia and Latin America. Toronto u. a.: University of Toronto Press.

Barry, James (1809): The Works. Bd. 1. London: Cadell \& Davies.

Bassani, Ezio (2000): African Art and Artefacts in European Collections 1400-1800. London: British Museum Press.

Basu, Priyanka (2012): „Die 'Anfänge' der Kunst und die Kunst der Naturvölker: Kunstwissenschaft um 1900“, in: Baleva, Martina/ Reichle, Ingeborg/Lerone Schultze, Oliver (Hrsg.): Image Match. Visueller Transfer, 'Imagescapes' und Intervisualität in globalen Bildkulturen. Paderborn/München: Fink, 109130.

Baudelot de Dairval, Charles-César (1686): L'utilité des voyages. 2 Bde. Paris: Aubouin \& Èmery.

Benzoni, Girolamo/Höniger, Nikolaus/Bry, Theodor de (1597): Neuwe und gründtliche Histoiren von dem nidergängischen Indien [...]. Bd. 3: Darinnen warhafftig erzehlet wirdt, wie die Spanier die Goldreichen Landschafften deß Peruanischen Königreichs eyngenommen [...]. Frankfurt a.M.: De Bry.

Besold, Christoph (1619): De natura populorum, eiusque pro loci positu, temporisque decursu variation. Tübingen: Cellius.

Borao Mateo, José E. (2010): „La version china de la obra ilustrada de Jerónimo Nadal Evangelicae Historiae Imagines", in: Goya 330/2, 16-33.

Bosc-Tiessé, Claire (2004): "The Use of Occidental Engravings in Ethiopian Painting in the $17^{\text {th }}$ and $18^{\text {th }}$ Centuries", in: João Ramos, Manuel/Boavida, Isabel (Hrsg.): The Indigenous and the Foreign in Christian Ethiopian Art. On Portugese-Ethiopian Contacts in the $16^{\text {th }}-17^{\text {th }}$ Centuries. Lissabon/Aldershot: Ashgate, 83-102.

Bostwick Davis, Elliot (2002): „Life Drawing from Ape to Human: Charles Darwin's Theories of Evolution and William Rimmer's Art Anatomy", in: Master Drawings 40, 345-359.

Bruer, Stephanie-Gerrit (2010): „Suche nach den Anfängen der Kunst. Idole in der Klassischen Archäologie im 18. und frühen 19. Jahrhundert", in: Kunze, Max (Hrsg.): Götzen, Götter und Idole. Frühe Menschenbilder aus 10 Jahrtausenden. Ruhpolding/Mainz: von Zabern, 79-86.

Buddensieg, Tilmann (1965): „Gregory the Great, the Destroyer of Pagan Idols: The History of a Legend Concerning the Decline of Ancient Art and Literature", in: Journal of the Warburg and Courtauld Institutes 28, 44-65.

Bujok, Elke (2004): Neue Welten in europäischen Sammlungen. Africana und Americana in Kunstkammern bis 1670. Berlin: Reimer.

Camille, Michael (1989): The Gothic Idol. Ideology and Image-Making in Medieval Art. Cambridge: Cambridge University Press. 
Caterino, Aldo (Hrsg.) (2008): Riflessi d'Oriente. Limmagine della Cina nella cartografia europea. Trient/Genua: Il Portolano.

Cavizzi, Joanne Antonio (1694): Historische Beschreibung der in dem untern Occidentalischen Mohrenland liegenden drey Königreichen/Congo, Matamba, und Angola. München: Jäcklin.

Chang, Sheng-Ching (2003): Natur und Landschaft: der Einfluss von Athanasius Kirchers "China illustrata" auf die europäische Kunst. Berlin: Reimer.

Cole, Michael W. (2009): „Perpetual Exorcism in Sistine Rome“, in: Cole, Michael W. / Zorach, Rebecca (Hrsg.): The Idol in the Age of Art, Farnham / Burlington (VT): Ashgate, 57-76.

Collet, Dominik (2007): Die Welt in der Stube. Begegnungen mit Außereuropa in Kunstkammern der Frühen Neuzeit. Göttingen: Vandenhoeck \& Ruprecht.

Connelly, Frances S. (1995): The Sleep of Reason. Primitivism in Modern European Art and Aesthetics 1725-1907. University Park: The Pennsylvania State University Press.

Cortez, Hernán (1986): Letters from Mexico. Hrsg. von Anthony Pagden. New Haven/London: Yale University Press.

Cousin, Louis (1688): La morale de Confucius. Amsterdam: Savouret.

Cummins, Thomas B.F. (2010): „Through the „Devil's Looking-glass“ Darkly: Brazilians, Peruvians, Aztecs, and Zemis in Europe; Serlio and Hercules in the Americas", in: Pierce, Donna (Hrsg.): The Arts of South America 1492-1850. Denver: Denver Art Museum, 11-38.

Deal, David M. / Hostetler, Laura (2006): The Art of Ethnography. A Chinese „Miao Album". Seattle/London: University of Washington Press.

Deswartes Rosa, Sylvie (1985): „Antiquité e nouveaux mondes. A propos de Francisco de Holanda", in: Revue de l'art 68, 55-72.

Diels, Hermann/Kranz, Walther (Hrsg.) (1960): Die Fragmente der Vorsokratiker. Berlin: Weidmann.

Effinger, Maria/Logemann, Cornelia/Pfisterer, Ulrich (Hrsg.) (2012): Götterbilder und Götzendiener in der Frühen Neuzeit. Europas Blick auf fremde Religionen. Heidelberg: Winter.

Fontana, Michela (2010): Matteo Ricci: gesuita scienziato umanista in Cina. Rom: De Luca.

Ginzburg, Carlo (1999): „Götzen und Abbilder. Die Wirkungsgeschichte eines Origines-Textes", in: ders.: Holzaugen. Über Nähe und Distanz. Berlin: Wagenbach, $144-167$.

Gisi, Lucas M. (2007): Einbildungskraft und Mythologie. Die Verschränkung von Anthropologie und Geschichte im 18. Jahrhundert. Berlin/New York: De Gruyter.

González de Mendoza, Juan (1598): Historien und Bericht von dem newlicher Zeit erfundenen Königreich China [...]. Leipzig: Schnelboltz.

Grimmelshausen, Johann Jakob Christoph (1977): Grimmelshausens Werke. Hrsg. von Siegfried Streller. Bd. 1. Berlin/Weimar: Aufbau.

Guevara, Felipe de (1948): Comentarios de la Pintura. Hrsg. von Rafael Benet. Barcelona: Selecciones bibliófilas (Impr. S.A.D.A.G.). 
Häfner, Ralph (2003): Götter im Exil. Frühneuzeitliches Dichtungsverständnis im Spannungsfeld christlicher Apologetik und philologischer Kritik (ca. 15901736). Tübingen: Niemeyer.

Harriot, Thomas/Bry, Theodor de (1590): Wunderbarliche, doch Warhafftige Erklärung, Von der Gelegenheit, und Sitten der Wilden in Virginia. Frankfurt a. M.: Feyerabend.

Haynes, Clare (2012): „In the Shadow of the Idol: Religion in British Art Theory, 1600-1800“, in: Art History 31, 63-85.

Himmelmann, Nikolaus (1986): Antike Götter im Mittelalter. Mainz: von Zabern.

Honour, Hugh (1976): The New Golden Land. European Images of America from the Discovery to the Present Time. London: Allen Lane.

Hoogstraten, Samuel van (1678): Inleyding tot de hooge schoole der schilderkonst. Rotterdam: van Hoogstraeten.

Hospinianus, Rudolphus ( $\left.{ }^{2} 1603\right)$ : De Templis. Zürich: Wolf.

Hostetler, Laura (2001): Quing Colonial Enterprise: Ethnography and Cartography in Early Modern China. Chicago: University of Chicago Press.

Hsia, Florence C. (2009): Sojouners in a Strange Land. Jesuits and Their Scientific Missions in Late Imperial China. Chicago: University of Chicago Press.

Hsia, Ronnier Po-chia (2010): A Jesuit in the Forbidden City: Matteo Ricci 15521610. Oxford: University of Oxford Press.

Iselin, Isaak ( $\left.{ }^{4} 1779\right)$ : Über die Geschichte der Menschheit. Basel: Schweighauser [Neudruck der Ausgabe 1764].

Jackson, Anna/ Jaffer, Amin (Hrsg.) (2004): Encounters. The Meeting of Asia and Europe 1500-1800. London: Victoria \& Albert Museum.

Jane, Cecil (1989) (Hrsg.): The Journal of Christopher Columbus. New York: Bonanza Books.

Jauß, Hans Robert (1964): „Ästhetische Normen und geschichtliche Reflexion in der 'Querelle des Anciens et des Modernes"“, in: Perrault, Charles: Parallèle des Anciens et des Modernes. Hrsg. von Hans Robert Jauß und Max Imdahl. München: Fink, 8-64.

Kaufmann, Thomas DaCosta (1996): „Eurocentrism and Art History? Universal History and the Historiography of the Arts before Winckelmann", in: Reinink, Wessel/Stumpel, Jeroen (Hrsg.): Memory \& Oblivion. Dordrecht: Kluwer Academic Publishers, 35-42.

Kircher, Athanasius (1667): China monumentis, qua sacra, qua profanis [...] illustrate. Amsterdam: Jan Janszoon van Waesberge \& Eliza Weyerstraet.

Klüser, Günter (2009): „Traditionelle chinesische Kartographie. Eine kritische Aufarbeitung absurder Darstellungen“, in: Michalsky, Tanja/Schneider, Felicitas / Engel, Gisela (Hrsg.): Aufsicht-Ansicht-Einsicht. Neue Perspektiven auf die Kartographie an der Schwelle zur Frühen Neuzeit. Berlin: Trafo, 21 - 42.

Kohl, Karl-Heinz (1986): Entzauberter Blick. Das Bild vom guten Wilden und die Erfahrung der Zivilisation. Frankfurt a. M.: Suhrkamp.

Kraus, Michael/ Ottomeyer, Hans (2007): Novos Mundos. Neue Welten. Portugal und das Zeitalter der Entdeckungen. Berlin/Dresden: Sandstein.

Kunze, Max (Hrsg.) (2010): Götzen, Götter und Idole. Frühe Menschenbilder aus 10 Jahrtausenden. Ruhpolding/Mainz: von Zabern. 
Lazure, Guy (2007): „Nadal au Nouveau Monde. Une traduction poétique des Evangelicae Historiae Imagines, Pérou, vers 1614", in: Dekoninck, Ralph/Guideroni-Bruslé, Agnès (Hrsg.): Emblemata Sacra. Rhétorique et herméneutique du discours sacré dans la literature en images. Turnhout: Brepols, $321-331$.

Lecercle, François (1990): „Des yeux pour ne point voir. L'idolâtrie dans la théologie des images au XVI ${ }^{\mathrm{e}}$ siècle“, in: Lidolâtrie. Paris: Editions L'Harmattan, 35-41.

Lecercle, François (2005): „L'obscénité de l'idole - à propos du Trattato della pittura e scultura, uso et abuso loro de G. D. Ottonelli et Pietro da Cortona (1652)“, in: Dekoninck, Ralph/Watthee-Delmotte, Myriam (Hrsg.): L'idole dans l'imaginaire occidental. Paris u. a.: L'Harmattan, 155-165.

Ledderose, Lothar (1991): „Chinese influence on European art - sixteenth to eighteenth century", in: Lee, Thomas H. C. (Hrsg.): China and EuropeImages and Influences in the Sixteenth to Eighteenth Centuries. Hong Kong: Chinese University Press, 221-249.

Lein, Edgar (2002): „Imagini degli dei indiani. La representación de las divinidades indianas por Vincenzo Cartari“, in: Kügelgen, Helga von (Hrsg.): Herencias indigenas, tradiciones europeas y la mirada europea. Frankfurt a.M. u. a.: Vervuert, $225-258$.

Loyer, Godefroy (1714): Relation du voyage du royaume d'Issyny, Paris: Arnoul Seneuze.

Lupher, David A. (2003): Romans in a New World. Classical Models in SixteenthCentury Spanish America. Ann Arbor. Michigan: University of Michigan Press.

MacCormack, Sabine (2006): "Gods, Demons, and Idols in the Andes“, in: Journal of the History of Ideas 67, 623-648.

Marin, Louis (2007): „Die Angst vor dem Idol“, in: ders.: Von den Mächten des Bildes. Zürich/Berlin: Diaphanes, 75-82. [Neudruck der franz. Ausgabe 1993]

Maspoli Genetelli, Silvia (2006): Il filosofo e le grottesche. La pluralità dell'esperienza estetica in Montaigne, Lomazzo e Bruno. Rom/ Padua: Antenore.

Miller, Isaac (2004): „Idolatry and the Polemics of World-Formation from Philo to Augustine", in: Journal of Religious History 28, 126-145.

Miller, Peter (2001): „Taking Paganism seriously: Anthropology and Antiquarianism in Early Seventeenth-Century Histories of Religion", in: Archiv für Religionsgeschichte 3, 183-209.

Mitter, Partha (1977): Much Maligned Monsters: History of European Reactions to Indian Art. Oxford: University of Chicago Press.

Mochizuki, Mia M. (2010): „The Moveable Center: The Netherlandish Map in Japan", in: North, Michael (Hrsg.): Artistic and Cultural Exchanges between Europe and Asia, 1400-1900. Rethinking Markets, Workshops and Collections, Farnham/Burlington, VT: Ashgate, 109-133.

Mulsow, Martin (2001): „John Seldens De Diis Syris. Idolatriekritik und vergleichende Religionsgeschichte im 17. Jahrhundert", in: Archiv für Religionsgeschichte 3, 1-24.

Mulsow, Martin (2005): „Antiquarianism and Idolatry. The historia of religions in the seventeenth century", in: Pomata, Gianna/Siraisi, Nancy (Hrsg.): His- 
toria, Empiricism and Erudition in Early Modern Europe. Cambridge / London: MIT Press, 181-209.

Mulsow, Martin (2012): ,Jean-François Lafitau und die Entdeckung der Religionsund Kulturvergleiche", in: Effinger, Maria/Logemann, Cornelia/Pfisterer, Ulrich (Hrsg.): Götterbilder und Götzendiener in der Frühen Neuzeit. Europas Blick auf fremde Religionen. Heidelberg: Winter, 37-47.

Mungello, David E. (1987): „Aus den Anfängen der Chinakunde in Europa 16871770", in: Walravens, Hartmut (Hrsg.): China illustrate. Das europäische Chinaverständnis im Spiegel des 16. bis 18. Jahrhunderts. Weinheim: VCH, 67-78.

Neuber, Wolfang (1995): „Exotismus, physiognomischer Blick und der Körper des 'Indianers' in der Frühen Neuzeit", in: Frühneuzeit-Info 6, 172-180.

Odell, Dawn (2009): „Creaturely Invented Letters and Dead Chinese Idols“, in: Cole, Michael/Zorach, Rebecca (Hrsg.): The Idol in the Age of Art. Farnham/Burlington (VT): Ashgate, 267-288.

Olanda, Francisco d' (2003): I Trattati d'Arte. Hrsg. von Grazia Modroni. Livorno: Sillabe.

Pagden, Anthony (1986): The Fall of Natural Man. The American Indian and the Origins of Comparative Ethnology. Cambridge: Cambridge University Press.

Perrault, Charles (1668): Parallele des anciens et des modernes. Paris/Amsterdam: Gallet.

Perrault, Charles (1690): Le Cabinet des beaux Arts. Paris: Edelinde.

Pfisterer, Ulrich (2008): „Origins and Principles of World Art History: 1900 (and 2000)", in: Zijlmans, Kitty/ Damme, Wilfried van (Hrsg.): World Art Studies: Exploring Concepts and Approaches. Amsterdam: Valiz, 69-89.

Pfisterer, Ulrich (2009): „'Der Kampf um's Weib’-oder: Kupka, Darwin und die Evolution der Kunst(-Geschichte)", in: Krass, Urte (Hrsg.): Was macht die Kunst? Aus der Werkstatt der Kunstgeschichte. München: Utz, 121-160.

Pfisterer, Ulrich (2011): „Animal Art/Human Art: Imagined Borderlines in the Renaissance “, in: Höfele, Andreas / Laqué, Stephan (Hrsg.): Humankinds. The Renaissance and Its Anthropologies. Berlin/New York: De Gruyter (= P \& A, 25), $217-246$.

Pietz, William (1983, 1987 und 1988): „The Problem of the Fetish“, in: RES 9, 5$17,13,23-45$ und $16,105-123$.

Pirazzoli-T'Serstevens, Michèle (2007): Giuseppe Castiglione 1688-1766. Peintre et architecte à la cour de Chine. Paris: Thalia.

Prescher, Nadine (2010): „Götzen und die Reformationszeit“, in: Kunze, Max (Hrsg.): Götzen, Götter und Idole. Frühe Menschenbilder aus 10 Jahrtausenden. Ruhpolding/Mainz: von Zabern, 9-24.

Purtle, Jennifer (2010): „Scopic Frames: Devices for Seeing China c. 1640“, in: Art History 33, 54-73.

Rees, Joachim (2012): „Vergleichende Verfahren - verfahrene Vergleiche. Kunstgeschichte als komperative Kunstwissenschaft - eine Problemskizze“, in: kritische berichte 40/2, 32-43.

Rogers, Francis M. (1962): The Quest for Eastern Christians: Travels and Rumor in the Age of Discovery. Minneapolis: University of Minnesota Press. 
Röttgen, Steffi (1998): „Begegnungen mit Apollo. Zur Rezeptionsgeschichte des Apollo von Belvedere im 18. Jahrhundert", in: Andreae, Bernard u. a. (Hrsg.): Il Cortile delle Statue. Der Statuenhof des Belvedere im Vatikan. Mainz: von Zabern, 253-274.

Rubiés, Joan-Pau (2006): „Theology, Ethnology, and the Historicization of Idolatry", in: Journal of the History of Ideas 67, 571-596.

Russo, Alessandra/ Wolf, Gerhard / Fane, Diana (2011): El vuelo e las imágenes. Arte plumaria en México y Europa. Mexiko City: Museo Nacional de Arte.

Sandrart, Joachim von (1675-1679): Teutsche Academie der edlen Bau-, Bild-und Mahlerey-Künste. 2 Bde. Nürnberg: Jacob von Sandrart.

Schmidt, Francis (1985): „Naissance des Polythéismes (1624-1757)“, in: Archives de Sciences Sociales des Religions 59, 77-90.

Schweizer, Anton u. a. (Hrsg.) (2011): Japanische Lackkunst für Bayerns Fürsten. München: Staatliche Münzsammlung.

Seznec, Jean (1931): „Un essai de mythologie comparée au début du XVII c siècle“, in: Mélanges d'Histoire et d'Archéologie, $268-281$.

Sölch, Brigitte (2007): Francesco Bianchini (1662-1729) und die Anfänge öffentlicher Museen in Rom. München/Berlin: Deutscher Kunstverlag.

Spielmann, Heinz/Drees, Jan (Hrsg.) (1997): Gottorf im Glanz des Barock. Bd. 2: Die Gottorfer Kunstkammer. Schleswig: Schleswig-Holsteinisches Landesmuseum.

Steadman, John M. (2006): „Eve's Dream and the Conventions of Witchcraft “, in: Journal of the History of Ideas 67, 567-574.

Tarde, Jean (1984): A la rencontre de Galilée. Deux voyages en Italie. Hrsg. von François Moreau und Marcel Tetel. Genf: Droz.

Trigault, Nicolas (1617): Historia von Einführung der christlichen Religion in das große Königreich China durch die Societet Jesu. Augsburg: Antonius Hierat von Cöllen.

Vespucci, Amerigo (1992): Letters from a New World. Hrsg. von Luciano Formisano. New York: Marsilio Pub.

Walravens, Hartmut (Hrsg.) (1987): China illustrate. Das europäische Chinaverständnis im Spiegel des 16. bis 18. Jahrhunderts. Weinheim: VCH.

Weinshenker, Anne B. (2008): A God or a Bench. Sculpture as a Problematic Art during the Ancien Régime. Bern u. a.: Lang.

Whitfield, Roderick (1976): „Chinese Paintings from the Collection of Archduke Ferdinand II", in: Oriental Art 22, 406-416.

Winckelmann, Johann J. ( $\left.{ }^{2} 1776\right)$ : Geschichte der Kunst des Alterthums. Wien: Wissenschaftliche Buchgesellschaft.

Wolf, Gerhard (2009): „Fluid borders, hybrid objects. Mediterranean art histories $500-1500$, questions of method and terminology", in: Anderson, Jaymie (Hrsg.): Crossing Cultures. Carlton: Miegunyah Press, 134-137.

Zedelmeier, Helmut (2003): Der Anfang der Geschichte. Studien zur Ursprungsdebatte im 18. Jahrhundert. Hamburg: Meiner.

Zelle, Carsten (1995): Die doppelte Ästhetik der Moderne: Revisionen des Schönen von Boileau bis Nietzsche. Stuttgart/Weimar: Metzler.

Zittel, Claus (2002): „'Truth is the daughter of time'. Zum Verhältnis von Theorie der Wissenskultur, Wissensideal, Methode und Wissensordnung bei Francis 
Bacon", in: Detel, Wolfgang/Zittel, Claus (Hrsg.): Ideals and Cultures of Knowledge in Early Modern Europe/Wissensideale und Wissenskulturen in der frühen Neuzeit. Berlin: Akademie-Verlag, 213-238.

\section{Abbildungsnachweise}

Abb. 1: URL: http://commons.wikimedia.org/wiki/File:Kunyu Wanguo Quantu (坤興萬國全圖).jpg

Abb. 2: Privatbesitz des Autors.

Abb. 3: UB Heidelberg.

Abb. 4: UB Heidelberg.

Abb. 5: UB Heidelberg.

Abb. 6: UB Heidelberg.

Abb. 7: Kunze 2010.

Abb. 8: Privatbesitz des Autors.

Abb. 9: UB Heidelberg.

Abb. 10: UB Heidelberg.

Abb. 11: Privatbesitz des Autors.

Abb. 12: UB Heidelberg. 